\title{
Quasi-Neutral Particle Simulation of Magnetized Plasma Discharges: General Formalism and Application to ECR Discharges
}

\author{
Martin Lampe, Member, IEEE, Glenn Joyce, Member, IEEE, \\ Wallace M. Manheimer, Fellow, IEEE, and Steven P. Slinker
}

\begin{abstract}
We have developed an electrostatic particle-incell/Monte Carlo (PIC/MC) simulation method for magnetized discharges, in which both internal electric fields and sheath potentials are determined from the requirement of quasineutrality within the bulk plasma, rather than by solving Poisson's equation. Thus the electric field is not sensitive to statistical noise which may occur in the small quantity $n_{e}-n_{i}$. Sheaths are treated self consistently as thin potential barriers, and the Bohm criterion for ion flux into the sheath is imposed as a boundary condition. Electron plasma oscillations do not appear in the model, and the debye length is essentially set to zero. Thus time steps and spatial gridding can be chosen to represent the characteristic macroscopic time and space scales of interest, which may be orders of magnitude larger than the plasma frequency/debye length scales. The simulation technique correctly represents kinetic features such as non-Maxwellian distributions and Landau damping and can be used for either collisional or collisionless plasmas. We present results from an axisymmetric simulation of an electron cyclotron resonance (ECR) discharge in low-pressure argon, which show that the discharge is strongly affected by cross-field ion flows, even when the vessel walls are insulators. We also present analytic calculations based on the model, which afford new insights into cross-field transport in a metallic vessel and show that the classic Simon diffusion can be strongly inhibited by the effect of sheath potentials.
\end{abstract}

Index Terms-Electron cyclotron resonance plasma, particlein-cell simulation, particle simulation, plasma simulation, quasineutral plasma.

\section{INTRODUCTION}

$\mathbf{I}$ $\mathrm{N}$ recent years, there has been considerable interest in the use of low pressure, high density plasma sources for materials processing, e.g., for semiconductor etching and also for some types of chemical vapor deposition [1]. These types of sources, which include electron cyclotron resonance (ECR), helicon, and inductively-coupled sources, operate at gas pressure ranging from 10 mtorr to below 1 mtorr and plasma density from $10^{11}$ to $10^{13} \mathrm{~cm}^{-3}$. In this regime, fluid models are not well matched to the characteristics of either the ions or the electrons. For example, mean free paths can be

Manuscript received July 1, 1997; revised June 4, 1998. This work was supported by the Office of Naval Research.

The authors are with the Plasma Physics Division, Naval Research Laboratory, Washington, DC 20375-5346 USA (e-mail: lampe@ppd.nrl.navy.mil; joyce@ppd.nrl.navy.mil; manheime@ccs.nrl.navy. mil; slinker@ppd.nrl.navy.mil).

Publisher Item Identifier S 0093-3813(98)09649-0. comparable to device dimensions, the electron response to the driving electromagnetic fields can be nonlocal, and velocity distribution functions can be nonMaxwellian (particularly for the high energy electrons that determine sheath potentials, ionization rates, and other inelastic collisional processes). For these reasons, fully kinetic simulation models are needed to properly represent the overall physics and chemistry of the discharge.

A natural approach to modeling of these types of discharges is the use of particle-in-cell (PIC) computer simulations which also include a Monte Carlo (MC) representation of collisional processes (PIC/MC models) [2]. In these simulations, the motion of particles between collisions is followed deterministically under the influence of specified external magnetic fields and self-consistent electrostatic fields computed by calculating the charge density of the particles and then solving Poisson's equation. Recent years have seen major advances in the development and use of PIC/MC codes [2]-[12]. In most cases, the objective of a simulation is to calculate the characteristics of the plasma steady state, or of a slowly varying quasi-steady state (scale time $>10^{-5} \mathrm{~s}$ ), as a function of the machine control parameters. However, the enormous range of spatial and temporal scales in a high-density discharge precludes straightforward application of the explicit PIC/MC procedure to multidimensional simulations of the entire discharge. As an example, the spatial and temporal scales for a typical ECR plasma are summarized in Table I. With respect to plasma transport and electrical properties, the approach to a steady state occurs over time scales characterized by the escape of ions to the walls, which are typically greater than $100 \mu \mathrm{s}$. The evolution to a steady state of the chemistry may occur on an even longer time scale characterized by the residence of neutrals in the system. However, in order to obey accuracy and stability criteria, a standard explicit PIC code must resolve all of the shorter time scales, down to and including the electron plasma frequency, electron gyrofrequency, and microwave frequency time scales, which are on the order of tens of picoseconds. Similarly, the spatial scales of real interest in a reactor-scale simulation are macroscopic scale lengths (centimeters), but a standard explicit particle simulation would have to resolve a vast range of spatial scales, down to the electron gyroradius, the debye length, and the thickness of passive sheaths, which are typically $0.001-0.1 \mathrm{~cm}$. It is not practical to resolve this range of spatial and temporal scales 
TABLE I

Typical Parameters For an ECR Discharge in ARgon

Microwave frequency $2.45 \times 10^{9} \mathrm{~Hz}$

Pressure 1 mTorr
Spatially-varying parameters:

Magnetic field 20 - 1000 Gauss

Plasma density $10^{11}-10^{13} / \mathrm{cm}^{3}$

Electron temperature $1-10 \mathrm{eV}$

\begin{tabular}{lrrlr}
\multicolumn{2}{c}{ Nominal Time Scales (sec) } & \multicolumn{2}{c}{ Nominal Spatial Scales (cm) } \\
Electron plasma oscillation & $0.6-6 \times 10^{-11}$ & Debye length & 0.001 \\
Electron gyration & $6-300 \times 10^{-11}$ & Electron gyroradius & 0.005 to 0.2 \\
Electron transit through ECR zone & $10^{-8}$ & Sheath thickness & 0.01 \\
Electron collision frequency & $10^{-7}$ & Electron mfp & 10 's \\
Ion plasma oscillation & $1.5-15 \times 10^{-9}$ & Microwave wavelength & $<12$ \\
Ion gyration & $4-200 \times 10^{-6}$ & Ion gyroradius & $0.01-2$ \\
Ion collision frequency & & $10^{-5}$ & Ion mfp & 10 \\
Ion lifetime & $10^{-4}$ & Device size & $10-100$ \\
Neutral residence time & $10^{-2}$ & & \\
\hline
\end{tabular}

within a multidimensional simulation. To the greatest extent possible, one would like to simulate the macroscopic features and the approach to equilibrium, while excluding the fast time scales and short spatial scales from the simulation.

Within the bulk plasma, it is possible to avoid resolution of the electron plasma frequency time scale and the debye length scale by using an implicit scheme to solve the Poisson equation together with the particle equations of motion [13]-[21]. In a properly framed implicit scheme, modes which are not resolved are damped away. However, the use of implicit PIC coding does not in itself resolve all of the problems. A first problem area is the sheath. In high-density discharges, sheaths are very narrow, and typically one may not need to resolve the sheath structure. However, it is absolutely essential to know the sheath potential (a spatially varying quantity), in order to determine the properties of the overall discharge. Therefore, a PIC/Poisson simulation, even if it is implicit, must resolve the debye-scale spatial structure and the associated plasma-frequency time scale in the sheath. It is possible to use a finer grid in the sheath and subcycle the time steps there, and this has been done in modeling of RF capacitive discharges [22]. However this procedure is quite complex, and to our knowledge has never been done within a multidimensional PIC-electron/PIC-ion code that simulates an entire high-density discharge over the entire approach to equilibrium.

A second problem is that it becomes increasingly difficult to implement implicit coding in the limit of time steps $\Delta t$ which are orders of magnitude longer than the plasma time and grid cell sizes $\Delta x$ which are orders of magnitude longer than $\lambda_{D}$. For example, Gibbons and Hewett [19] find that energy conservation over long times requires that the cell size be related to the time step and the electron thermal velocity $v_{e}$ by $\Delta x / \Delta t \simeq 3 v_{e}$, and that this relation must be satisfied with increasing accuracy as $\omega_{p} \Delta t$ increases. Cohen et al. [20] reach a similar conclusion, but in their code the energy conservation condition is $\Delta x / \Delta t \simeq v_{e}$. The model dependence of the energy conservation condition is discussed in [19]. In a simulation where $\omega_{p}, \lambda_{D}$, and $v_{e}$ show strong spatial or temporal variation, it may be difficult to satisfy the energy conservation condition over the whole simulation.

A third problem area occurs in applying PIC/Poisson simulation techniques to quiescent quasi-neutral plasmas, such as processing discharges. In these plasmas, the electrostatic potential varies by only a few times the electron temperature $T_{e}$ within the bulk plasma, giving rise to a weak electric field $\boldsymbol{E}$ (less than $1 \mathrm{~V} / \mathrm{cm}$ up to a few $\mathrm{V} / \mathrm{cm}$ ). However, this electrostatic field controls transport of plasma toward the walls and therefore strongly influences the overall structure and characteristics of the discharge. When viewed in the light of Poisson's equation

$$
\nabla^{2} \phi=4 \pi e\left(n_{e}-n_{i}\right)
$$

the source of this field $n_{i}-n_{e}$ represents an electron-ion fractional charge separation, over a scale length $R$ that is of order $10 \lambda_{D}^{2} / R^{2}$. This difference $n_{i}-n_{e}$, which must be determined from the simulation particle positions with accuracy and low noise, is typically less than $10^{-\tilde{5}}$ of the density of either electrons or ions $\left(\lambda_{D} \simeq 0.001 \mathrm{~cm}, R>1\right.$ $\mathrm{cm}$ ).

For all of these reasons, numerical schemes involving Poisson's equation are obviously very difficult in the quasineutral limit. In this limit, we would argue that the more natural point of view is that the electrostatic field (and, as we shall see, the sheath potential as well) take the value that is necessary to maintain quasi-neutrality within the bulk plasma, and the charge separation takes the value that is necessary to maintain this electric field. This viewpoint is to be contrasted with the conventional view that the electric field is "determined" by the charge separation through Poisson's equation. Indeed, Chen [23] commented long ago that, "In a plasma, it is usually possible to assume $n_{e}=n_{i}$ and $\nabla \cdot \boldsymbol{E} \neq 0$ 
at the same time. This is a fundamental trait of plasmas, one which is difficult for the novice to understand. Do not use Poisson's equation to obtain $\boldsymbol{E}$ unless it is unavoidable!"

Over the years, this approach has been followed in many analytic and numerical models which represent the plasma as a fluid and in hybrid schemes which represent the electrons as a fluid and the ions as particles [24]-[32]. In the simplest implementation, these methods circumvent the use of Poisson's equation by neglecting electron inertia, assuming constant $T_{e}$, and determining $\boldsymbol{E}$ from the Boltzmann relation. The ion density $n_{i}$ is then determined from the dynamical equations, and $n_{e}$ is simply set equal to $n_{i}$ to maintain quasineutrality. In more complex treatments [10], [32], electron inertia is retained and $\boldsymbol{E}$ is determined from the electron and ion continuity, momentum, and possibly energy conservation equations, together with the quasi-neutrality relation. These procedures eliminate temporal scales on the order of the electron plasma frequency, as well as spatial structures on the debye length scale. Fluid or hybrid models of this type are the technique of choice for quasi-neutral plasmas whenever an isothermal fluid-electron formulation is acceptable. Fluid models have been used for many years in simulation of RF discharges; for these discharges, the pressure is usually high enough for fluid assumptions to be generally reasonable. In recent years, hybrid fluid electron/particle ion techniques have been applied to the simulation of ECR plasmas [8]-[11], in some cases [8], [11] with the full use of Poisson's equation and therefore resolution of the sheath, and in other cases [9], [10] with analytic sheath models and/or quasi-neutrality assumptions in the bulk. The authors of [8]-[11] indicate that the fluid-electron assumption is made only for convenience and is not expected to be valid. For example, in [11] the electron density for use as the source term in Poisson's equation is determined from the Boltzmann relation, assuming $T_{e}$ constant along a magnetic field line. ${ }^{1}$ However, particle electrons are also pushed in the fields that are thus determined, and the spatially-resolved electron velocity distribution is determined from the particles. The results show that the electron distribution is very far from Maxwell-Boltzmann, and indeed that $T_{e}$ has strong spatial variation, so that the assumptions of the model are invalid.

Thus it appears that a full kinetic description of all species, e.g., a PIC-electron/PIC-ion code, is necessary for an ECR discharge, and this is true for many other situations where the bulk plasma is quasi-neutral. We report here on the development of a new fully kinetic approach to the analysis and simulation of bounded, weakly collisional plasma discharges in which the bulk plasma is quasi-neutral. Electrostatic fields within

\footnotetext{
${ }^{1}$ In [11], the very brief presentation of the computational model may leave the impression that the electric field is determined by implicit solution of the Poisson equation with the charge density determined by the PIC electrons and PIC ions. However, in the more extensive presentation of the model given by K. A. Ashtiani, "A 2-D particle-in-cell (PIC) model of an axisymmetric electron-cyclotron-resonance (ECR) plasma-processing system," Ph.D. dissertation, University of Wisconsin-Madison, Dept. of Electrical and Computer Engineering, 1995, (4.7) shows that in the solution of Poisson's equation the PIC electrons are actually replaced by a Boltzmann distribution part of the time. Thus the model is actually a hybrid model, with the PIC electrons serving only as test particles which determine $T_{e}$ and (nonself-consistently) determine the electron distribution function.
}

the bulk plasma, as well as sheath potentials, are computed in a self-consistent spatially and temporally resolved way, but Poisson's equation is not used. ${ }^{2}$ At the present stage, the model specifically treats axisymmetric situations in which the electrons are strongly magnetized in a specified nonuniform magnetic field, as in ECR plasmas, but we believe that by combining the methods used here with those discussed in [32], the general approach can be extended to three dimensions and used for unmagnetized or weakly magnetized cases. The approach is also applicable to quasi-neutral processes in high-temperature collisionless plasma. In the numerical implementation, both electrons and ions are represented as PIC particles, with Monte Carlo collisions. At present, neutrals are assumed to form a uniform background, but neutrals could be treated as another simulation species with MC collisions. The spatial gridding reflects the macroscopic scale lengths, and the time steps are chosen to resolve particle motion over macroscopic lengths. We shall also give some examples in which the model provides a natural framework for analytic calculations, leading to new insights. The model has the following characteristics.

1) Electrons are assumed to be strongly magnetized, and thus are treated as guiding center particles, firmly attached to a single flux surface. This eliminates the electron gyro time scale from the dynamics. However, the ion gyro motion is resolved both spatially and temporally, and ions are pushed through their orbits under the influence of the electrostatic and Lorentz forces (as well as collisions).

2) The electric field component $E_{\|}$, parallel to the magnetic field lines, is determined by the requirement that $E_{\|}$ drive the electrons to maintain quasi-neutrality. This field can be specified by a slightly modified form of the electron parallel momentum conservation equation with neglect of electron inertia.

3) Sheaths, at either grounded or floating surfaces, are regarded as thin potential barriers to electron flow. The sheath width is set to zero, but the sheath potentials are determined self-consistently by the requirement that electron flow to walls be consistent with preservation of quasi-neutrality in the plasma and also with constraints on current flow to the walls.

4) The Bohm condition on ion flow at the bulk-sheath interface is imposed as a boundary condition on the bulk flow. This is a key aspect of the model, since the Bohm condition is the principal constraint driving plasma flow to the walls. The quasi-neutral presheath is resolved within the model.

5) The relative plasma potential on different magnetic field lines, and therefore the electric field component $E_{\perp}$ transverse to the magnetic field, is determined through conditions on the transverse ion flow necessary to maintain quasi-neutrality. The transverse electric field and the transverse ion flow depend significantly on whether the field lines terminate on insulating or con-

\footnotetext{
${ }^{2}$ To the best of our knowledge, quasi-neutrality has not previously been used to determine $E$ in a full-PIC code. In [32], Hewett outlines a procedure for using quasi-neutrality to calculate $\mathrm{E}$, but implements it only in a hybrid model.
} 
ducting walls. In the case of conducting walls, the sheath potentials and the transverse electric field are intimately related and must be determined self consistently. In this paper, we present the formalism for both cases and show simulation results for the insulating case, which is considerably simpler to implement.

6) Monte Carlo electron-neutral and ion-neutral collisions are included, and a highly efficient Langevin/Monte Carlo method [33] is used to include electron-electron and ion-ion Coulomb collisions as well. ${ }^{3}$ Since the plasma density is high, Coulomb collisions (which have been omitted in most previous models of processing plasmas) play an important role, e.g., in Maxwellianizing the tail of the electron distribution function and in isotropizing the ion distribution.

7) Resonant heating of the plasma by the microwaves can also be represented in a self-consistent quasi-linear formulation which eliminates short time scales such as the gyro period and is structurally similar to the Monte Carlo technique used for electron-electron collisions [34]. However, this aspect will be implemented in a future publication.

Within this framework, plasma oscillations are excluded from the model. Simulation time steps need not resolve the electron plasma or gyro frequency; thus they can be chosen to resolve motion of individual particles over macroscopic lengths of interest and/or collisional time scales of interest. In typical cases (e.g., Ar plasma at pressure $<2$ mtorr), we use ion time steps of order few $\times 10^{-7} \mathrm{~s}$ and sub-cycled electron time steps of order $10^{-8} \mathrm{~s}$. The result is a very efficient code that can be run to equilibration (time scales of order $300 \mu \mathrm{s}$ ) in about five hours on a $180 \mathrm{MHz}$ Pentium Pro PC. The selfconsistent separation of sheath and bulk plasma also facilitates intuitive understanding and provides a useful framework for analytic calculations. For example, in Section VII we shall see how self-consistent variations of the sheath potential can strongly inhibit cross-field ion transport.

\section{Structure And GeOMetry OF The Model}

To help motivate the assumptions of the model, it may be useful to consider some typical plasma conditions which are of interest to us (while keeping in mind that the model is applicable to a much broader range of conditions). In ECR processing sources, the microwave frequency is usually $2.45 \mathrm{GHz}$, so that electron gyroresonance occurs at $875 \mathrm{G}$. In a two-solenoid source (Fig. 1), the magnetic field lines typically diverge downstream, so that $B \sim 1000 \mathrm{G}$ where the microwaves are introduced, decreases to the resonant value nearby, and falls to as low as $20 \mathrm{G}$ far downstream. The electron temperature $T_{e}$ is typically a few $\mathrm{eV}$. The ion temperature $T_{i}$ is typically much lower, but the ion flow speed reaches the ion sound speed $c_{s} \equiv\left(T_{e} / m_{i}\right)^{1 / 2}$ at the interface between the bulk plasma and the sheath. Many gas compositions are used for processing, and pressure may vary

\footnotetext{
${ }^{3}$ Electron-ion collisions are also easily treated using the formulation in Manheimer et al., but they are not included in the simulation shown in Section VIII of this paper.
}

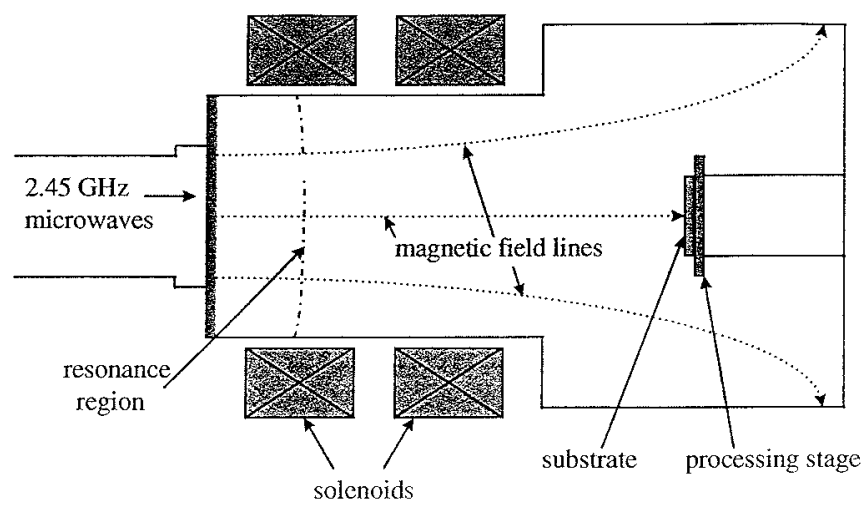

Fig. 1. Schematic drawing of a typical two-magnet ECR plasma reactor.

from 0.1 mtorr to $>10$ mtorr, but for specificity we shall consider Ar at pressure 1 mtorr. Typically, the plasma density $n$ will be in the range $10^{11}<n<10^{13} \mathrm{~cm}^{-3}$ so that the ionization fraction is roughly $1-20 \%$.

Under these conditions, the electron gyroradius is extremely small ( $\ll 1 \mathrm{~mm}$ in most of the device) and the electron mean free path is so long (about $40 \mathrm{~cm}$ for electron-neutral collisions [35], [36] and $30 \mathrm{~cm}$ for electron-ion collisions at plasma density $10^{12} \mathrm{~cm}^{-3}$ and $T_{e}=4 \mathrm{eV}$ ) that electron collisional diffusion across field lines is very slow. Typically an electron will diffuse across field lines by $<1 \mathrm{~cm}$, before eventually escaping from the system to a wall. Thus, it is quite reasonable to neglect cross-field collisional electron diffusion entirely, even though electron collisions are included in the model and have important effects on the electron velocity distribution. Furthermore, we shall restrict our attention in this paper to axisymmetric systems, so that all electron drifts are azimuthal. Thus, we may assume that each electron is strictly confined to a particular flux tube. ${ }^{4}$ In the framework of $r$ - $z$ geometry, where the azimuthal coordinate is ignorable, we may think of the electrons as strung out along the magnetic field lines, with the electron position specified by a single coordinate along the field line.

In order to treat the electron dynamics efficiently and accurately, we use magnetic field lines, denoted by a discrete index $j$, as one of the coordinates for our grid. The spacing of the field lines chosen for the grid is arbitrary, and in principle may be chosen to optimize the resolution in regions of particular interest. However, there are also numerical constraints on the choice of grid field lines, e.g., it is necessary to maintain an adequate number of particles on each grid field line to control statistical fluctuations. The grid used in typical simulations is shown in Fig. 2. The axial coordinate $z$ is used as the other coordinate for the grid; equally spaced grid lines in $z$ are denoted by the index $k$. The use of a nonuniform and nonorthogonal grid, which also intercepts the radial wall at oblique angles, complicates the code structure considerably, but affords great simplicity and efficiency in the treatment

\footnotetext{
${ }^{4}$ Since azimuthal drift velocities play no role in any of the considerations of this paper, we shall consistently use the notation $v_{\perp}$ to refer to the electron gyration speed, not including any drift. If it is necessary to calculate azimuthal electron drifts, this can be done within the framework of the model. Also, it is possible to include electron cross-field transport as a slow collisional diffusion, but this will not be discussed further in this paper.
} 


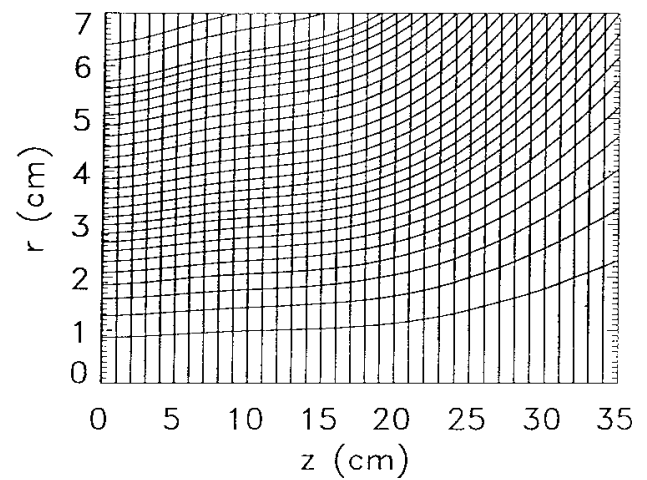

Fig. 2. In the simulation shown in this paper, the geometry of the ECR source is simplified to a cylinder. The simulation grid, shown in this figure, is formed by a chosen set of magnetic field lines (the actual measured field lines in an experimental configuration) and by an equally spaced $z$ grid.

of electron dynamics: each electron is permanently associated with a single field line $j$ during its entire lifetime in the system and in the particle lay-down is linearly distributed onto the two nearest grid points $(j, k)$ and $(j, k+1)$.

The ion gyroradius ranges from a few $\mathrm{mm}$ for thermal ions in the strong field region, to tens of $\mathrm{cm}$ for ions moving at $c_{s}$ in the weak field region. Furthermore, the ion mean free path for charge exchange [37]-[39] $(\sim 10 \mathrm{~cm})$ is often comparable to or moderately larger than the ion gyroradius. ${ }^{5}$ Thus the ions are only weakly magnetized. In the model, the ion orbits are computed in full 3-D, under the influence of the externally specified magnetic field, the self-consistent electric fields which are interpolated from the field line grid onto an $r$ $z$ grid, and Monte Carlo collisions. After the completion of an ion time step, the ion density is laid down on the $j-k$ grid, as follows. Each ion is attributed in $z$ fully to its nearest neighbor grid value $k$, and then is apportioned among the two field lines between which it is located, according to the quadratic formula

$$
\begin{aligned}
\eta_{j k} & =\frac{r_{j+1, k}^{2}-r^{2}}{r_{j+1, k}^{2}-r_{j k}^{2}} \\
\eta_{j+1, k} & =\frac{r^{2}-r_{j k}^{2}}{r_{j+1, k}^{2}-r_{j k}^{2}}
\end{aligned}
$$

which is appropriate to $r$ - $z$ geometry. Here, the ion coordinate $r$ is located between field lines $j$ and $j+1$, with $r_{j k}$ and $r_{j+1, k}$ the coordinates of the nearest grid points on field lines $j$ and $j+1$ respectively, and $\eta_{j k}$ is the fraction of the ion that is attributed to field line $j$.

\section{Electron Dynamics AND PARAllel Electric FiEld}

Since the electrons are represented as guiding center particles, each electron is characterized by its location along the field line. In the code we use the Cartesian coordinate $z$ to specify this location, but in analytic development it is often useful to use $\zeta$, the curvilinear coordinate along the field line. In addition, each electron has a parallel velocity $v_{\|}$and a perpendicular (gyrating) velocity $v_{\perp} .{ }^{4}$ We assume that the

\footnotetext{
${ }^{5}$ Note that the total cross section for elastic ion-neutral scattering is comparable to the charge exchange cross section [38], but elastic scattering is predominantly at very small angles [39].
}

magnetic moment $\mu \equiv m_{e} v_{\perp}^{2} / 2|B|$ is a constant of the motion in between collisions, so usually it is convenient to characterize the electron by its value of $\mu$ rather than $v_{\perp}$. The effective parallel force acting on an electron, between collisions, is then

$$
F_{\|}=-e E_{\|}-\mu(d|B| d \zeta)
$$

where the second term is the mirror force [23]. In the computational model, the electrons are pushed, between collisions, as simulation particles subject to the force $F_{\|}$. The time step $\Delta t_{e}$ for the electron push is chosen to resolve electron motion over macroscopic lengths of interest and also to resolve electron collisional time scales. Typically $\Delta t_{e}$ is a small fraction of the time step $\Delta t_{i}$ used to push ions.

Let us assume for the moment that the number of electrons on a field line $j$ is equal to the number of ions assigned to that field line, so that the field line is globally quasi-neutral. (We will later discuss how to insure that this is true.) The electron debye length is always smaller than any scale length resolved in the model, and the electron plasma frequency is always fast compared to any time scale resolved in the model. Thus, if there were any departure from local quasi-neutrality, the resulting strong electric field would drive electron currents parallel to $\boldsymbol{B}$, which would restore quasi-neutrality within a time scale of several electron plasma periods, i.e., essentially instantaneously on the time scale of the model. Thus, the macroscopic parallel electric field always takes the value necessary to keep the electron density $n_{e}$ equal to the ion density $n_{i}$. To specify this electric field, we can begin with the electron momentum equation. For magnetized electrons on curved field lines, this equation takes the form

$$
\begin{aligned}
-e E_{\|}= & \frac{|B|}{n_{e}} \frac{\partial}{\partial \zeta} \frac{\left(P_{e \|}+n_{e} m_{e} u_{e \|}^{2}\right)}{|B|}+\bar{\mu} \frac{\partial|B|}{\partial \zeta} \\
& +\nu_{e} m_{e} u_{e \|}+\frac{1}{n_{e}} \frac{\partial}{\partial t}\left(n_{e} m_{e} u_{e \|}\right) .
\end{aligned}
$$

Here, $m_{e}$ is the electron mass, $P_{e \|} \equiv n_{e} T_{e \|}$ is the electron parallel pressure

$$
P_{e \|} \equiv \int d v_{\|} m_{e}\left(v_{\|}-u_{e \|}\right)^{2} f_{e}\left(v_{\|}, v_{\perp}\right)
$$

$u_{e \|}$ is the mean electron fluid velocity, $\bar{\mu}$ is the average magnetic moment at the specified location, and $\nu_{e}$ is the mean electron momentum transfer collision frequency. The derivation of (4) is outlined in the Appendix.

Within the particle simulation, the first three terms on the right-hand side (RHS) of (4) can be evaluated at each point of the grid by laying down the mean quantities for the electrons assigned to that grid point. These terms represent the "ambipolar" electric field necessary to sustain the quasineutrality relation

$$
n_{e}=n_{i}
$$

if $n_{i}$ were a specified time-independent function of $\zeta$. The remaining term (the inertial term) is a small correction: because of the quasi-neutrality constraint, $n_{e}=n_{i}$, the electron flow velocity $u_{e}$ is of comparable magnitude to the ion flow velocity $u_{i}$, and the time derivative is on the ion time scale; 
hence this term is typically of order $m_{e} / m_{i}$, where $m_{i}$ is the ion mass. In quasi-neutral fluid formulations, where $n_{e}$ is simply set equal to $n_{i}$, the inertial term is neglected. However this is unsatisfactory in a particle simulation, where $n_{e}$ and $n_{i}$ are separately determined by the particle evolution: if the inertial term is neglected, $n_{e}$ and $n_{i}$ will drift apart as $n_{i}$ slowly changes and $n_{e}$ does not respond. Even worse, in a particle code both $n_{e}$ and $n_{i}$ are subject to continual fluctuations, and the ambipolar field allows $n_{e}$ and $n_{i}$ to separate through these fluctuations.

There are several possible approaches to the calculation of the inertial term, or of some approximate form that couples $n_{e}$ to $n_{i}$. In a previous publication [40], we developed an approximate method in which the electrons are pushed in the ambipolar field, and then at the end of the time step a correction field is applied that is chosen to restore quasineutrality. Although this method worked well in most respects, we have found another approach that is even simpler and very accurately conserves energy over long time scales. We simply drop the inertial term in (4) and substitute $n_{i}$ for $n_{e}$ in the first term of (4), so that

$$
\begin{aligned}
-e E_{\|}= & \frac{|B|}{n_{i}} \frac{\partial}{\partial \zeta} \frac{\left[n_{i}\left(T_{e \|}+m_{e} u_{e \|}^{2}\right)\right]}{|B|} \\
& +\bar{\mu} \frac{\partial|B|}{\partial \zeta}+\nu_{e} m_{e} u_{e \|} \cdot
\end{aligned}
$$

Equation (7) keeps the electron density closely coupled to the ion density, while avoiding the very high-frequency plasma oscillations that are linked to the last term of (4). This can be seen by substituting (7) for $E_{\|}$in the exact momentum (4), to obtain 6

$$
m_{e} \frac{\partial}{\partial t}\left(n_{e} u_{e \|}\right)=n_{e}\left(T_{e}+m u_{e \|}^{2}\right) \frac{\partial}{\partial \zeta} \ln \left(\frac{n_{i}}{n_{e}}\right) .
$$

Equation (8) shows that the electrons are always accelerated up the gradient in $n_{i} / n_{e}$, i.e., toward the point of maximum positive net charge density, thereby restoring quasi-neutrality. We showed in [41] that this scheme is stable, preserves $n_{e}=n_{i}$ while supporting only lower-frequency oscillations, conserves energy to good accuracy, and accurately represents kinetic effects. For example, the usual dispersion relation for ion sound waves, including the Landau damping terms, can be derived [41]. We also gave several examples of successful implementation of the scheme in a one-dimensional (1-D) particle simulation with no boundaries, including linear and nonlinear ion sound waves and free expansion of a plasma [41].

\section{Sheaths AT Passive SuRfaces}

At any material surface in contact with the plasma, there is a sheath where quasi-neutrality fails. We shall consider here only sheaths at unpowered or weakly dc-biased surfaces, e.g., vessel walls. The sheath thickness is typically a few times the debye length $\lambda_{D}$, which is very thin for conditions of interest.

\footnotetext{
${ }^{6}$ Equation (8) differs slightly from the corresponding (7) of our previous paper [41]. We are grateful to Dr. M. E. Riley for pointing out a minor error in the latter equation, which we have corrected here.
}

For example, $\lambda_{D}=0.0015 \mathrm{~cm}$ at $T_{e}=4 \mathrm{eV}, n=10^{12} \mathrm{~cm}^{-3}$. Such a sheath can be treated as simply a thin potential jump $\phi_{s}$, which accelerates positive ions toward the wall, but reflects most electrons back into the plasma. At any given time, only a few electrons with high enough parallel energy can surmount the sheath potential barrier and reach the wall. (However, any electron can eventually diffuse up to high energy, due to ECR heating or electron-electron collisions, and escape from the system.) The effect of the sheath on the plasma, or on the surface, is essentially completely characterized by the sheath potential.

To determine the value of the sheath potential, we use an elaboration of the "logical sheath" scheme of Parker, Procassini, Birdsall and Cohen [42]. Our formulation incorporates the Bohm flow criterion, applies appropriately to both conducting and insulating walls, and can be used for a multidimensional plasma with magnetized electrons. The basic idea is that the sheath potential takes the value which allows the "correct" electron flux to reach the wall. The conditions for determining the correct flux depend on whether the wall is conducting or insulating.

\section{A. Insulating Walls}

An insulating wall exposed to a plasma acquires a surface charge during an initial transient period (which we do not resolve), and thereafter the electrical current density to any point on the wall must be zero. Thus the sheath potential $\phi_{S}$ at each end of field line $j$ must take the value (the floating potential) which sets the flux of electrons, through the sheath to the wall, equal to the ion flux into the sheath from the plasma. (All positive ions which reach the boundary of the simulation pass through the sheath and reach the wall.) Furthermore, according to the Bohm criterion [43], [44] ${ }^{7}$ ions must flow from the bulk plasma into the sheath at a mean velocity equal to the ion sound speed $c_{s}$, i.e., the ion flux to any wall is

$$
J_{i}^{\mathrm{Bohm}}=n c_{s} .
$$

In the next section we shall discuss the way in which the Bohm criterion is imposed as a boundary condition on the ion flow in the model. However, the implication for the electrons is that the electron flux to any wall $J_{e}^{\text {out }}$ must satisfy ${ }^{8}$

$$
J_{e}^{\text {out }}=\sin \theta \int_{v_{s}}^{\infty} d v_{\|} v_{\|} f_{e}\left(v_{\|}\right)=J_{i}^{\text {Bohm }}
$$

where

$$
v_{s} \equiv\left(2 e \phi_{s} / m_{e}\right)^{1 / 2}
$$

${ }^{7}$ For simplicity, in this paper we use the form of the Bohm criterion (16) which holds when the ion temperature is small. For warm ions, a more complex form of the Bohm criterion holds, as discussed in [43]. Using the techniques discussed in this paper, it is possible to enforce the more accurate form of the Bohm condition, and the effects of this can be significant in some situations.

${ }^{8}$ Equations (10) and (13) for $J_{e}^{\text {out }}$ assume that the guiding center electron model can be used in the sheath as well as the bulk. For situations where a field line intersects the wall at glancing incidence, and where the electron gyroradius exceeds the sheath width, a more elaborate model is needed which includes gyro motion. Essentially, the $\sin \theta$ factor is weakened and dependence on $v_{\perp}$ is introduced. 
and $\theta$ is the angle between the magnetic field and the wall. If the electron velocity distribution function $f_{e}\left(v_{\|}\right)$at the end of field line $j$ is known, (10) and (11) determine the sheath potential $\phi_{s}$.

To implement this prescription numerically, we first push both the electrons and ions through a complete ion time step $\Delta t_{i}$. If an electron reaches a wall, it is reflected back along its field line, on the tentative assumption that its energy was insufficient to penetrate the sheath potential barrier. However, it is noted that this particular electron belongs to the set

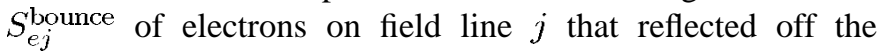
wall during $\Delta t_{i}$. In general, this number of electrons will be much larger than the number of ions that reach that wall during the time step, $\Delta N_{i j}^{\text {Bohm }} \equiv J_{i}^{\text {Bohm }} \Delta t_{i} A_{j}$ where $A_{j}$ is the area intercepted on the wall by the flux tube associated with field line $j$. We then identify the subset $S_{e j}^{\text {out }}$ of $S_{e j}^{\text {bounce }}$, consisting of the $\Delta N_{i j}^{\text {Bohm }}$ electrons which had the highest parallel energy at the moment of reflection. These electrons are assumed to have escaped during the time step; hence they are discarded. The sheath potential $\phi_{s j}$ is set equal to the lowest parallel kinetic energy of any of the electrons in the subset $S_{e j}^{\text {out }}$ at the time it reached the simulation boundary.

There are a number of numerical points which must be considered in actually implementing this scheme. The sheath potential depends on the high-energy tail of the electron distribution, which always contains relatively few simulation electrons and is susceptible to noise. To minimize this, $\Delta t_{i}$ must be large enough that a substantial absolute number of electrons are allowed to escape during the time step. However, the fraction of electrons which leave during $\Delta t_{i}$ must be small. In a steady state situation, the departed electrons will be replaced by new electrons generated by ionizing collisions, and one must be careful not to overcount ionizing collisions involving electrons that actually escaped during $\Delta t_{i}$. Also, there may be electrons with high parallel velocities that bounce off both walls during the time step $\Delta t_{i}$, and these must be accounted for correctly.

\section{B. Conducting Walls}

When the walls are conducting, there will typically be a boundary condition specifying the potential on any given segment of the wall. In the simplest case, the walls may all be regarded as grounded, but in other cases a dc bias may be applied. (We do not consider ac biases in this paper.) For conducting walls, there is a more complicated set of conditions that determines the sheath potentials. It is possible for nonzero electrical current density to flow to any given point on the wall, i.e., the electron flux to the wall need not be locally equal to the ion flux out of the plasma. Within the plasma, there may be currents flowing along a field line, and also ion currents flowing across field lines. However, quasi-neutrality imposes a constraint on the electron and ion fluxes: the total number of ions and electrons on any given field line must remain equal. This is the condition that determines the sheath potentials.

Let $J_{e j}^{\text {out }}(0)$ be the electron flux to the wall at the $z=0$ end of field line $j$ and $J_{e j}^{\text {out }}\left(z_{\max }\right)$ be the flux to the wall at the other end of field line $j$. Let $J_{i j}^{\text {Bohm }}(0)$ and $J_{i j}^{\text {Bohm }}\left(z_{\max }\right)$ be the Bohm flux of ions into the sheaths at $z=0$ and $z_{\max }$, respectively. Then maintenance of global quasi-neutrality on field line $j$ requires that

$$
\begin{aligned}
& J_{e j}^{\text {out }}(0)+J_{e j}^{\text {out }}\left(z_{\max }\right)= \\
& \quad J_{i j}^{\text {Bohm }}(0)+J_{i j}^{\text {Bohm }}\left(z_{\max }\right)+\int_{0}^{z_{\max }} d z \nabla \cdot J_{i \perp j}(z) .
\end{aligned}
$$

The last term in (12) represents the net decrease in the number of ions attributed to field line $j$ due to ion flow across field lines within the plasma.

In (12), the electron fluxes to the walls ${ }^{8}$ are determined by the sheath potentials $\phi_{s j}(0)$ and $\phi_{s j}\left(z_{\max }\right)$

$$
\begin{aligned}
& J_{e j}^{\text {out }}(z=0)=\sin \theta_{j 0} \int_{-\infty}^{-v_{s j}(z=0)} d v_{\|} v_{\|} f_{e}\left(z=0, v_{\|}\right) \\
& J_{e j}^{\text {out }}\left(z_{\max }\right)=\sin \theta_{j, \max } \int_{v_{s j}\left(z_{\max }\right)}^{\infty} d v_{\|} v_{\|} f_{e}\left(z_{\max }, v_{\|}\right)
\end{aligned}
$$

where

$$
\begin{aligned}
v_{s j}(z=0) & \equiv\left[2 e \phi_{s j}(z=0) / m_{e}\right]^{1 / 2} \\
v_{s j}\left(z_{\max }\right) & \equiv\left[2 e \phi_{s j}\left(z_{\max }\right) / m_{e}\right]^{1 / 2}
\end{aligned}
$$

$\theta_{j 0}$ is the angle between field line $j$ and the wall at $z=0$, and $\theta_{j, \max }$ the angle at $z=z_{\max }$. If the walls are grounded $(\phi=$ 0 ), then the plasma potential just inside the sheath, at either $z=0$ or $z_{\max }$, is just equal to the sheath potential. However, the difference between the plasma potentials $\phi_{j}\left(z_{\max }\right)$ and $\phi_{j}(0)$ is given by the line integral of $E_{\|}(z)$, the parallel electric field given by (7). Thus the two sheath potentials are related by

$$
\phi_{s j}\left(z_{\max }\right)-\phi_{s j}(0)=-\int_{0}^{z_{\max }} d z E_{\|}(z)
$$

and (12) in effect specifies both sheath potentials, provided the electron energy distributions and the ion flux are known from the simulation.

If there is an externally imposed potential difference along field line $j$, between the walls at $z=0$ and $z_{\max }$, then (14) takes the more general form

$$
\phi_{s j}\left(z_{\max }\right)-\phi_{s j}(0)=\phi_{\mathrm{ext}}-\int_{0}^{z_{\max }} d z E_{\|}(z)
$$

where $\phi_{\text {ext }}$ is the imposed potential difference. While (12) specifies the total electron flux escaping from the field line, (15), together with the rest of the simulation equations, determines separately the flux of electrons to the walls at $z=0$ and $z_{\max }$, and thus in effect the electric current flowing along the field line. In this way, the simulation calculates the electric current density in terms of the imposed voltage, i.e., it gives a macroscopic Ohm's law for the plasma.

It is clear from this formulation that in the case of conducting walls the sheath potentials are intimately connected to the cross-field ion flow. We shall see that it is necessary to calculate both the sheath potentials and the ion flow selfconsistently at each time step. Therefore, we shall defer the 
discussion of a scheme for actually calculating the sheath potentials to Section VI where the ion dynamics are discussed.

\section{IMPOSITION OF THE BOHM \\ CRITERION AS A BOUNDARY CONDITION}

Whenever a plasma comes into contact with a material wall, a sheath forms immediately as a response to the rapid loss of electrons to the wall. At the same time, the resulting electrostatic field also penetrates into the quasi-neutral bulk plasma and accelerates ions toward the wall. At the interface between the quasi-neutral bulk plasma and the ion-rich sheath, the component of the ion fluid velocity normal to the wall reaches a value given by ${ }^{7}$

$$
u_{i}^{\perp}=c_{s}
$$

if $T_{i} \ll T_{e}$. Indeed, it is well known that a steady-state monotonic sheath, with $n_{i}>n_{e}$, can form only where the ion flow in the bulk plasma satisfies (16). This is known as the Bohm criterion ${ }^{7}$ [1], [43], [44]. Note that in (16), we use the notation $u_{i}^{\perp}$ with superscript $\perp$ to denote the velocity normal to the wall, not to the magnetic field.

In a simulation model which properly resolves the sheath as well as the bulk plasma, and calculates the internal electric field through Poisson's equation, the Bohm condition will be satisfied automatically as the sheath forms. However, our quasi-neutral model, as described in the preceding sections, resolves only the bulk plasma and simply prescribes the existence of a sheath with the proper sheath potential. This boundary condition is incomplete: it does not automatically induce the appropriate bulk flow conditions which allow a sheath to form. Steady state solutions exist which satisfy (16), but there are also solutions which exhibit other ion flow patterns at the walls. For example, if the ions are initially cold and uniform and the electrons are isothermal throughout the bulk plasma, the electron pressure will remain uniform, no electric field will be generated, and there will be no ion flow toward the wall. Thus, within our model, the Bohm condition must be imposed externally as a boundary condition to the ion flow, which is necessary to specify the correct solution of the equations. One may say, from the perspective of the bulk plasma, that the Bohm condition causes the flow of the plasma toward the walls.

Within the context of a fluid model of the quasi-neutral bulk plasma, (16) can be imposed as a boundary condition to the ion momentum equation. But how is one to impose (16) as a boundary condition within a particle simulation? We have used a procedure that seems to imitate the dynamical process that sustains the Bohm condition within the quasi-neutral bulk part of a discharge, where the ion flow speed is equal to $c_{s}$ at the edge of the bulk plasma, but is much smaller within the interior of the plasma. The continuity equation thus implies that $n_{i}$ falls off at the edge of the bulk plasma. Quasi-neutrality requires that $n_{e}=n_{i} \equiv n$. Assuming $T_{e}$ does not vary as rapidly as $n$ at the plasma edge, the fall off in electron pressure at the edge leads to an ambipolar electric field [first term of (7)] which sustains the ion flow in the "presheath" region. In the model, we define a thin strip of depth $d$, adjacent to any material surface, with $d$ chosen to be macroscopically small but larger than $c_{s} \Delta t_{i}$. Within the strip, we lay down the ion velocities to determine the average ion flow speed $u_{i j}^{\perp}$ normal to the surface, on field line $j$. We then add an increment $\delta u_{i j}^{\perp}$ to all of the ion velocities in the strip, such as to bring $u_{i j}^{\perp}$ to $c_{s}$. This procedure insures that $n_{i}$ falls off at the perimeter of the bulk plasma just as it would if Bohm flow were occurring. The resulting pressure drop at the perimeter leads (over a time scale characterized by the ratio of the presheath width to $c_{s}$ ) to the formation of an appropriate presheath. Bohm flow is thereafter self-sustaining, but only neutrally stable, with the increment $\delta u_{i j}^{\perp}$ a small (positive or negative) adjustment at each time step.

The Bohm flow condition, together with the sheath potential as specified in Section IV, together represent a complete set of boundary conditions for the quasi-neutral bulk plasma. We shall refer to this as the "Bohm logical sheath" procedure.

\section{TRAnSVERSE EleCtric Field AND ION Transport ACross Magnetic FiEld Lines}

In Section III we showed how to determine the parallel electric field $E_{||}$from the requirements of quasi-neutrality, as applied to the electron flow along field lines. Thus, the relative potential between any two points on the same field line is known. If the absolute potential were specified at any single reference point on each field line, say at $z=0$, then it would be known everywhere, and the transverse electric field $E_{\perp}$ could also be computed. This additional information can also be obtained from the requirements of quasi-neutrality, but it is bound up with the ion flow rather than the electron flow. The conclusions also depend fundamentally on whether the field lines end on insulating walls or on grounded conducting walls.

\section{A. Insulating Walls}

In the case of insulating walls, electrical current cannot flow from the plasma to any point on the walls. Furthermore, in our model no transverse electron current is permitted within the plasma. It follows that if there is nonzero net ion flux onto any field line within the plasma, a violation of quasi-neutrality will occur on that field line. In a real plasma, a large potential would immediately build up all along the field line, leading to a reversal of the net transverse ion flow onto the field line, and subsequently to a train of ion plasma oscillations. To model this effect within a quasi-neutral framework, we shall stipulate that the integrated net ion flux onto any field line is zero, i.e.,

$$
\int d z \nabla_{\perp} \cdot\left(n \boldsymbol{u}_{i}\right)=0 .
$$

Equation (17), well satisfied on time scales longer than the ion plasma period, expresses the condition of quasi-neutrality and can be used to solve for the reference potential $\phi(z=0)$, on each field line.

To implement (17) numerically, we begin with the known electric field at the previous time step $t^{m}$, assume that quasineutrality was satisfied at $t^{m}$, and that the flux condition (17) was satisfied at the previous half-time step $t^{m-1 / 2}$. First, we push each ion to a new velocity $v^{*}$ at time $t^{m+1 / 2}$, using the 
electric fields determined at $t^{m}$, and to a new position $\left(r^{*}, z^{*}\right)$ at time $t^{m+1}$. At the end of the time step, there may be a small inequality of order $\Delta t_{i}^{2}$ between the number of ions $N_{i j}^{*}$ and electrons $N_{e j}^{*}$ on field line $j$, since the flux continuity condition is satisfied exactly at time $t^{m-1 / 2}$, but may be inaccurate by order $\Delta t_{i}$ during the time step

$$
\Delta N_{j}^{*} \equiv N_{i j}^{*}-N_{e j}^{*} \neq 0 \quad\left(\text { order } \Delta t_{i}^{2}\right) .
$$

This is an indication that a correction potential $\delta \phi_{j}$ should have been added to the assumed potential on field line $j$ so as to maintain the flux continuity (17) throughout the time step. This may be thought of as the correction to the reference potential $\phi_{j}(z=0)$. The corresponding correction to the radial electric field is

$$
\delta E_{r, j k}=\left(\delta \phi_{j}-\delta \phi_{j+1}\right) /\left(r_{j+1, k}-r_{j k}\right)
$$

at $z$-grid point $k$ between field lines $j$ and $j+1$. This in turn leads to a correction velocity

$$
\delta v_{r, j k}=\left(e \delta E_{r, j k} / m_{i}\right) \Delta t_{i}
$$

and a position displacement

$$
\delta r_{j k}=\left(e \delta E_{r, j k} / m_{i}\right) \Delta t_{i}^{2}
$$

for every ion in this grid cell. Using (19)-(21), the resulting correction to the number of ions on field line $j$ can then be written as

$$
\delta N_{i \perp j}=a_{j-1} \delta \phi_{j-1}-\left(a_{j}+a_{j-1}\right) \delta \phi_{j}+a_{j} \delta \phi_{j+1}
$$

where the coefficients $a_{j}$ are determined by first summing up the displacements of the particles, enumerated with the index $n$, which are attributed to $z$-grid point $k$ and lie between field lines $j$ and $(j+1)$, and then summing up the contributions of each grid point $k$ to the total number of ions on field line $j$. Given that the ions are apportioned among adjacent field lines according to the quadratic scheme (2), the coefficients are found to $\mathrm{be}^{9}$

$$
\begin{aligned}
a_{j}= & \frac{e \Delta t_{i}^{2}}{m_{i}} \sum_{k} \frac{1}{\left(r_{j+1, k}^{2}-r_{j k}^{2}\right)\left(r_{j+1, k}-r_{j k}\right)} \\
& \cdot \sum_{n}\left[2 r_{n}+\left(\frac{d r^{2}}{d z}\right)_{j k}\left(\frac{d r}{d z}\right)_{j k}\right]
\end{aligned}
$$

where $r_{n}$ is the coordinate of particle $n,(d r / d z)_{j k}$ is the slope of the magnetic field line at grid point $j k$, and $\left(d r^{2} / d z\right)_{j k} \equiv$ $2 r_{j k}(d r / d z)_{j k}$. The quantity $\delta N_{i \perp j}$ from (22) is then set equal to $-\Delta N_{j}^{*}$ so that the quasi-neutrality condition becomes

$$
a_{j-1} \delta \phi_{j-1}-\left(a_{j}+a_{j-1}\right) \delta \phi_{j}+a_{j} \delta \phi_{j+1}=-\Delta N_{j}^{*} .
$$

Equation (24) is a linear ordinary difference equation which determines $\delta \phi_{j}$, the correction to the potential $\phi_{j}(z=0)$

\footnotetext{
${ }^{9}$ When the magnetic field lines are oblique, the correction potentials $\delta \phi_{j}$ also lead to a perturbation to $v_{z}$, which makes an additional contribution to $\delta N_{i \perp j}$; this appears as the last term of (23).
}

which is necessary to maintain global quasi-neutrality. ${ }^{10}$ In this way, the potential difference between field lines is specified. After $\delta \phi_{j}$ has been calculated, the ions are pushed again in the corrected fields. Each field line $j$ will then have $N_{i j}=N_{e j}$ at time $t^{m+1}$, and (17) will be satisfied at $t^{m+1 / 2}$.

It will be noted that this procedure determines the plasma potentials and the ion flow in a coupled self-consistent way. However, the sheath potentials and the electron flow play no role in this determination. As we shall see, the situation is different when the walls of the vessel are conducting; in this case, the sheath potentials and the escape of electrons to the walls must also be included self consistently in the calculation.

\section{B. Grounded Walls}

In the case of grounded walls, the wall serves as a zero point for the potential that is common to all field lines. The potential in the bulk plasma on field line $j$, just inside the sheath at $z=0$, is thus equal to the sheath potential $\phi_{s j}$. If $\phi_{s j}$ is known, the potential can be calculated at any point on the field line, and the transverse E field can then be calculated by taking differences of potentials on adjacent field lines. However, the sheath potential is determined ${ }^{8}$ from (12)-(14), which involve the ion transverse flow. Thus the transverse ion flow is coupled to the escape of electrons through the sheaths.

To numerically implement the solution to this coupled problem, let us begin by rewriting (12) in the discrete form

$$
\Delta N_{e j}^{\text {out }}=\Delta N_{i j}^{\text {Bolm }}-\Delta N_{i \perp j} .
$$

Here $\Delta N_{e j}^{\text {out }}$ is the total number of electrons on field line $j$ that escape to the walls, through the sheaths at both ends, during time $\Delta t_{i}$. For any given electron energy distribution, $\Delta N_{e j}^{\text {out }}$ is a strong nonlinear function of $\phi_{s j} . \Delta N_{i j}^{\text {Bohm }}$ is the total number of ions that escape to the walls from flux tube $j$ during time $\Delta t_{i} . \Delta N_{i \perp j}$ is the net change in the number of ions attributed to field line $j$ due to transverse ion flow within the plasma.

The numerical solution of (25) is similar in spirit to the approach used for the case of insulating walls, but much more complicated in practice. Let us assume that the plasma was quasi-neutral at the previous time step $t^{m}$, that the sheath potentials $\phi_{s j}^{m}$ are known at $t^{m}$, and that (25) was satisfied at that time. We can then push the ions to new velocities $v^{*}$ at $t^{m+1 / 2}$ and positions $\left(r^{*}, z^{*}\right)$ at $t^{m+1}$ using the potentials known at time $t^{m}$. From the new ion positions, we can calculate a provisional value of $\Delta N_{i \perp j}$, which we shall call $\Delta N_{i \perp j}^{*} . \Delta N_{i j}^{\text {Bohm }}$ is slowly varying and can be evaluated at the beginning of the time step. However, in general,(25) will not be exactly satisfied at the end of the new time step (again the error will be of order $\Delta t_{i}^{2}$ ) because the ion and electron distributions in phase space will have changed a little since the previous time step, and the flux condition (12) will have been in error by order $\Delta t_{i}$.

\footnotetext{
${ }^{10}$ For the case in which the discharge is contained wholly within insulating walls, there is no way to specify the plasma potential with respect to the outside world. The ion flow depends only on the relative potential between adjacent field lines, and thus the quasi-neutrality condition should specify all but one of the potentials $\phi_{j}$. Indeed, it can be seen that (24) are redundant, and can be solved for all but one of the potentials.
} 
This is indicative that an increment $\delta \phi_{s j}$ should have been added to the sheath potentials $\phi_{s j}$ so as to preserve the flux condition throughout the time step and the quasi-neutrality condition at the end of the time step. In accordance with the reasoning that led to (22), this leads to a correction $\delta N_{i \perp j}$ to $\Delta N_{i \perp j}$, given by

$$
\delta N_{i \perp j}=a_{j-1} \delta \phi_{s, j-1}-\left(a_{j}+a_{j-1}\right) \delta \phi_{s j}+a_{j} \delta \phi_{s, j+1}
$$

where the coefficients $a_{j}$ are again given by (23). Now (25) can be written as

$$
\begin{aligned}
& a_{j-1} \delta \phi_{s, j-1}-\left(a_{j}+a_{j-1}\right) \delta \phi_{s j}+a_{j} \delta \phi_{s, j+1} \\
& \quad+\Delta N_{e j}^{\text {out }}\left(\phi_{s j}^{m}+\delta \phi_{s j}\right)=\Delta N_{i j}^{\text {Bohm }}-\Delta N_{i \perp j}^{*} .
\end{aligned}
$$

The RHS of (27) can be regarded as known, so (27) is an ordinary difference equation that determines the increments to the sheath potential $\delta \phi_{s j}$. The equation is strongly nonlinear through the term $\Delta N_{e j}^{\text {out }}$. Once the $\delta \phi_{s j}$ are known, the ions are pushed again in the newly determined potentials, and the electrons with energy exceeding the sheath potential are allowed to escape to the wall, as discussed in Section IV. Thus the computation can proceed, with the ion dynamics, the sheath potentials, and the transverse electric fields computed self-consistently at each time step.

The reader may have noticed that the calculation of the sheath potential correction $\delta \phi_{s j}$ is performed fully implicitly in conjunction with the particle push. This is in fact necessary for numerical stability: as will be seen in the next section, the sheath potential exerts a diffusive effect on transverse ion current, and the natural time step for this diffusion is shorter than we wish to resolve. Everything else in the computation is done explicitly.

\section{NATURE OF ION TRANSPORT}

\section{A. Insulating Walls: Field-Aligned Flow Model}

Section VI-A makes it clear that net ion transport across field lines is impossible in an insulating vessel, since the plasma must remain quasi-neutral and electron transport across field lines is negligible. However, it is quite possible for there to be a nonzero transverse ion flux at particular locations along a field line. Any transverse flux at one location must be canceled out by a reverse flux elsewhere along the field line. Thus, in situations where there are strong variations along a field line (as in Fig. 2 where the field lines flare outward downstream) one may find patterns of cross-field ion eddy flow. In the next section we shall see an example of this type of behavior in our simulations.

Nevertheless, we shall consider here a simple solvable 1-D fluid approximation, in which the ion flow is assumed to be field-aligned. This model, which extends the work of Godyak and collaborators [45], [46] to the case of flow along curved magnetic field lines, helps to interpret the two-dimensional (2D) simulations, and provides insight into the way in which the plasma temperature, density, and other properties are determined by the quasi-neutrality condition, the ambipolar potential, the Bohm boundary condition, and the requirement of global power balance. In the model, the electrons on any single field line are represented as an isothermal fluid. The ions are treated as a cold fluid, with field-aligned flow velocity $u_{i}$, subject to an electric field $E_{\|}=-\partial \phi / \partial \zeta$ and a constant mean free path $\lambda_{i}$ for (predominantly charge exchange ${ }^{5}$ [38], [39]) collisions with stationary neutrals. In addition, we assume that electron-impact ionization proceeds at frequency $\nu_{I}$, a function of $T_{e}$. In steady state, the ion continuity and momentum conservation equations, and the Boltzmann relation

$$
e[\phi-\phi(\zeta=0)]-T_{e} \ln [n / n(\zeta=0)]
$$

can be reduced to a single ordinary differential equation for the normalized ion flow velocity $u \equiv u_{i} / c_{s}$

$$
\left(1-u^{2}\right) \frac{d u}{d s}-\frac{u^{2}|u|}{\lambda}-u \frac{d \ln B}{d s}=\nu
$$

and a quadrature for the normalized density

$$
\frac{n(s)}{n(0)}=\exp \left[-\int_{0}^{s} d s^{\prime} u\left(\frac{|u|}{\lambda}+\frac{d u}{d s^{\prime}}\right)\right] .
$$

In (29) and (30), $s \equiv \zeta / \zeta_{\max }, \nu \equiv \zeta_{\max } \nu_{1} / c_{s}$, and $\lambda \equiv$ $\lambda_{i} / \zeta_{\max }$, where $\zeta$ is the curvilinear coordinate along the field line and $\zeta=0, \zeta=\zeta_{\max }$ are the points at which the field line intersects the vessel walls.

The boundary conditions for (29) arise from the Bohm condition, but some discussion is needed as to the proper way to apply the Bohm condition within a model of 1-D ion flow along field lines. If the field line intersects the walls normally, the appropriate boundary conditions on the fieldaligned velocity $u_{i}$ are given directly by the Bohm condition as stated in (16)

$$
\begin{aligned}
u_{i}(0) & =-c_{s} \\
u_{i}\left(\zeta_{\max }\right) & =+c_{s} .
\end{aligned}
$$

But if the field line intersects the walls obliquely, at angles $\theta_{0}$ and $\theta_{\max }$ at $\zeta=0$ and $\zeta_{\max }$, the appropriate boundary conditions at the sheath edge are (16) for the velocity component normal to the wall. To the extent that flow within the quasi-neutral plasma is strictly field-aligned, this would then give

$$
\begin{aligned}
u_{i}(0) & =-c_{s} / \sin \theta_{0} \\
u_{i}\left(\zeta_{\max }\right) & =+c_{s} / \sin \theta_{\max } .
\end{aligned}
$$

However, (32) are clearly not acceptable boundary conditions, since (29) becomes singular when $|u|=1$. The resolution to this dilemma is that 1-D flow along field lines is not a consistent model, even within the quasi-neutral plasma, at a point where the field lines intersect a wall obliquely. There is always a pre-sheath structure ${ }^{11}$ [47], beginning about where (31) are satisfied, wherein the ions are accelerated toward the wall and the density falls off by a factor of order $\sin \theta$. Thus the consistent boundary conditions, within the region where one can use a model of field-aligned ion flow, are indeed (31).

The two boundary conditions (31) applied to the single first-order ordinary differential (29) are an indication that (29)

\footnotetext{
${ }^{11}$ For example, in the case of a plasma with well-magnetized ions, it is known [48] that there is a pre-sheath structure, on the scale length of the ion Larmor radius, where the ion flow cannot be treated as field-aligned.
} 
and (31) together constitute a nonlinear eigenvalue problem: the ionization coefficient $\nu_{I}$ must take the value necessary to permit a solution. Since $\nu_{I}$ is a rapidly increasing function of $T_{e},(29)$ and (31) actually determine the discharge temperature, as well as the velocity profile $u_{i}(\zeta)$. The normalized density profile $n(\zeta) / n(0)$ and the normalized potential $[\phi(\zeta)-$ $\phi(0)] / T_{e}$ are then determined by (30) and (28). The absolute plasma density is determined by the power balance

$$
\begin{aligned}
W_{\text {in }}= & W_{\text {out }}=\left[n(0) c_{s} A_{\perp}(0)+n\left(\zeta_{\max }\right) c_{s} A_{\perp}\left(\zeta_{\max }\right)\right] \\
& \cdot\left(\epsilon_{I}+e \phi_{s}+\frac{5}{2} T_{e}\right)
\end{aligned}
$$

where $W_{\text {in }}$ is the microwave power input to the flux tube, $W_{\text {out }}$ is the energy loss rate due to electrons and ions reaching the walls, and $A_{\perp}(\zeta)$ is the cross-sectional area of the flux tube at $\zeta$. For Maxwellian electrons and cold ions, the energy loss per escaped ion is the sum of $\epsilon_{I}$, the electron inelastic collision energy loss per ionization (including excitation, dissociation, etc., as well as ionization); $e \phi_{s}+T_{e} / 2$, the ion energy at the wall; and $2 T_{e}$, the mean thermal energy of an escaping electron.

Equations (28)-(31) show that the normalized profiles $u_{i}(\zeta) / c_{s}, n(\zeta) / n(0)$ and $[\phi(\zeta)-\phi(0)] / T_{e}$ depend only on the collisionality parameter $\lambda$ and the shape of the magnetic field lines (but not the magnitude of the magnetic field). The temperature $T_{e}$ is determined by the requirement that the ionization rate be just sufficient to replace the ions lost to the walls. Since the eigenvalue $\nu$ is $\zeta_{\max } \nu_{I} / c_{s}$, the ionization rate $\nu_{I}$ is inversely dependent on the field line length $\zeta_{\max }$. In more physical terms, ions escape more rapidly from shorter field lines, and thus the ionization rate (i.e., $T_{e}$ ) must be larger to replace the lost ions. The density must be lower on these field lines so that a specified level of microwave power, divided among fewer electrons, is sufficient to raise $T_{e}$ to the required value.

In Fig. 3, we show a solution of (28)-(31) for a case with $\lambda=0.27, \zeta_{\max }=35 \mathrm{~cm}$, and

$$
B=B_{0} \exp \left[-\frac{\zeta^{2}}{(26.9 \mid \mathrm{cm})^{2}}\right]
$$

corresponding roughly to one of the long interior field lines of Fig. 2, with the gas being argon at pressure 1 mtorr. A strong left-right asymmetry is evident, introduced by the nonuniform magnetic field. At $z=0$, the ions are accelerated in a standard presheath of thickness $\sim \lambda_{i}$, but to the right the ions are accelerated by an electrostatic force arising from the mirror effect, and the plasma density falls off as the field lines diverge. Thus there is less need on the right for presheath acceleration to satisfy the Bohm condition, and indeed the presheath is less evident.

In Fig. 4, we show how the electron temperature $T_{e}$ on a particular field line depends on $\zeta_{\max }$, the length of that field line, with $B(\zeta)$ given by (34). Referring to the geometry shown in Fig. 2, we note that $\zeta_{\max }$ is essentially the same for all the interior field lines that terminate on the end wall, but decreases steadily as we go to the outer field lines that terminate on the radial wall. Thus the 1-D model predicts that

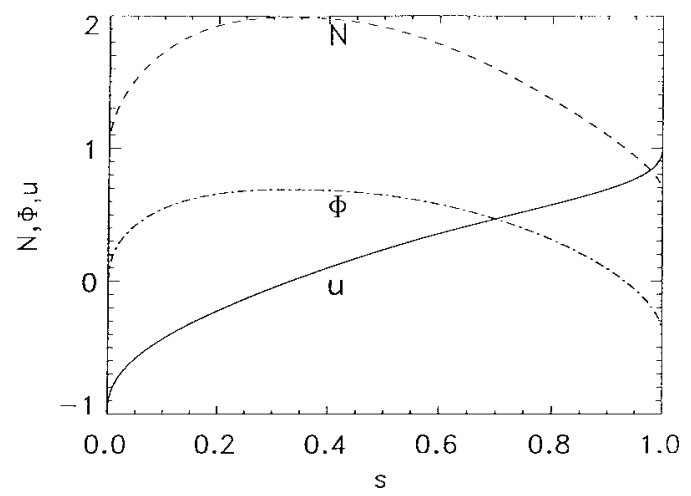

Fig. 3. Plasma state obtained by solution of the 1-D field-aligned flow model (28)-(31) for conditions approximating the long field lines of Fig. 2. Shown are the normalized density $N(s) \equiv n(s) / n(0)$ (dashed curve), the normalized ion flow velocity $u(s) \equiv u_{i}(s) / c_{s}$ (solid curve), and the normalized plasma potential $\Phi(s) \equiv[\phi(s)-\phi(0)] / T_{e}$ (dot-dashed curve). The eigenvalue is $\nu=1.56$ corresponding to $T_{e}=3.4 \mathrm{eV}$.

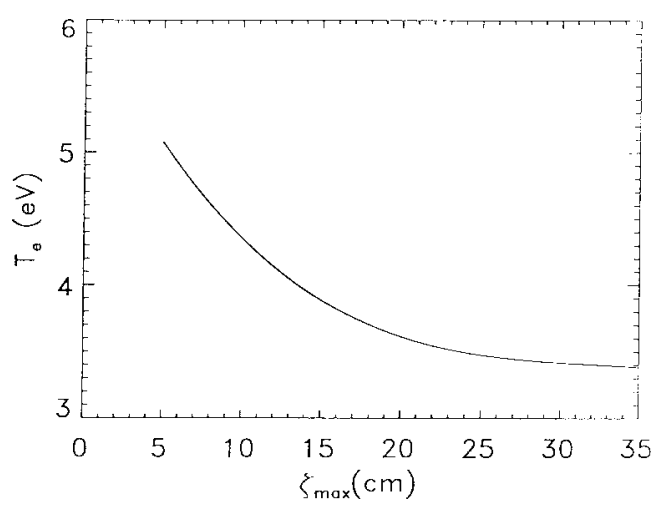

Fig. 4. Te as a function of field line length $\zeta_{\max }$, from the 1-D field-aligned flow model of Section VII-A, for conditions approximating the magnetic field configuration of Fig. 2.

$T_{e}$ will be constant on the interior field lines, but will steadily increase on the outer field lines. Similarly, the average density $\langle n\rangle$ on a particular field line is predicted to be constant on the interior lines but steadily decreasing on the outer field lines that terminate on the radial wall. The 1-D model of (28)-(31) and (33) has no dependence on the angles $\theta_{0}$ and $\theta_{\max }$, so there is no discontinuity in plasma properties between the last field line that terminates (nearly normally) on the end wall and the first that terminates (at glancing incidence) on the radial wall. As we shall see in Section VIII, this is not a realistic representation of the 2-D flow. In the 2-D simulation, field lines which intersect the radial wall lose ions much more rapidly, and thus $T_{e}$ is larger and the average density $\langle n\rangle$ is smaller for field lines that terminate on the radial wall.

We note that the rate at which ions escape from a field line is proportional to the ratio of the density at the walls to the mean density on the field line $\eta \equiv\left[n_{j}(0)+n_{j}\left(\zeta_{\max }\right)\right] / 2\left\langle n_{j}\right\rangle$. For this reason, $T_{e}$ and $\left\langle n_{j}\right\rangle$ are sensitive to the density profile $n_{j}(\zeta) / n_{j}(0)$ along the field line. Within our 1-D model, $\eta$ is determined by the solution of (29)-(31), but in our 2-D simulations other effects enter to change the density profile and thereby also the mean values $T_{e j}$ and $\left\langle n_{j}\right\rangle$. 


\section{B. Cross-Field Transport in Grounded Conducting Vessels}

In the case of grounded conducting walls, Simon [48]-[50] showed in 1955 that ions can diffuse across magnetic field lines at a rate characterized by the ion gyroradius and mean free path without dragging the electrons across field lines. Quasi-neutrality is maintained by electron flow to the walls along field lines. These calculations have been refined over the years [49], and in the present context of weakly magnetized ions and strong electric fields required to satisfy the Bohm condition, correspond to mobility-limited or inertially limited transverse ion flow [50]. However, the effect of the spatial variation of the sheath potential, induced by the ion flow, has traditionally been omitted from these models. Our quasineutral formulation of the sheath-coupled transverse flow lends itself to an exploration of this effect, which turns out to be dominant in the case of internal perturbations. The sheath potential strongly inhibits nonuniformities in the divergence of the transverse ion flow, as can be seen from the following considerations. Suppose that momentarily the flow is such as to increase the number of ions on field line $j$ more rapidly than the number of ions on adjacent field lines. To maintain global quasi-neutrality on field line $j$, it is necessary to reduce the electron flow to the walls from field line $j$ in accordance with (25). Therefore, the sheath potential on field line $j$ increases. But this also increases the plasma potential everywhere along field line $j$ and thus opposes the net ion transverse flow onto the field line. To gain analytic insight into this effect, we shall consider some very simplified model problems chosen to elucidate particular aspects of the problem.

Linearized Fluid Treatment: Consider a plasma contained within grounded walls at $z=0$ and $z=L$, but unbounded in the $x$ and $y$ directions. Let the magnetic field $\boldsymbol{B}_{z}$ be uniform and along the $z$ direction. Since we wish to focus on the effect of sheath potentials, which are normally very large compared to potential variations within the bulk plasma, we shall assume the bulk plasma in equilibrium is uniform with density $n_{o}$, plasma potential $\phi_{o}$ (equal to the sheath potential), and temperatures $T_{e}$ and $T_{i}$. However, we recognize the existence of a presheath at the walls by allowing the density at the walls $n_{w}$ to be smaller than $n_{o}$ by a factor $\eta$. We then consider a small deviation from equilibrium, with perturbations $n(x), \phi(x)$, and $u_{x}(x)$ to the density, sheath potential, and ion flow velocity, each of the form $e^{i k x}$. Assume for convenience that the perturbation maintains the isothermal character of the plasma and also the ratio $\eta$ of wall to bulk density.

We begin with the linearized ion momentum equation, which takes the form

$$
n_{o} \frac{\partial u_{x}}{\partial t}+\frac{i k P_{i}}{m_{i}}+\frac{e n_{o}}{m_{i}} i k \phi+\nu_{i}^{\prime} n_{o} u_{x}=0
$$

where

$$
\nu_{i}^{\prime} \equiv \nu_{i}\left(1+\frac{\Omega_{i}^{2}}{\nu_{i}^{2}}\right)
$$

$\nu_{i}$ is the ion-neutral momentum transfer collision frequency, and the second term in (36) results from the magnetization of the ions, i.e., from the $\boldsymbol{v}_{y} \times \boldsymbol{B}_{z}$ force. (This form is appropriate when $\partial u_{y} / \partial t \ll \nu_{i}$.)

We can relate $\phi$ to $n$ by linearizing (12). Defining a normalized distribution $F_{e}\left(v_{z}\right)$ by $f_{e}\left(v_{z}\right)=\left(n_{o}+n\right) F_{e}\left(v_{z}\right)$, the perturbation to the total electron flux to the two walls is

$$
\begin{aligned}
J_{e}^{\text {out }}= & 2 \eta n \int_{\left(2 e \phi_{o} / m_{e}\right)^{1 / 2}}^{\infty} d v_{z} v_{z} F_{e}\left(z_{z}\right) \\
& -\frac{2 \eta n_{o}}{m_{e}} F_{e}\left[\left(\frac{2 e \phi_{o}}{m_{e}}\right)^{1 / 2}\right] e \phi .
\end{aligned}
$$

The perturbation to the ion flux to the two walls is

$$
J_{i}^{\mathrm{Bohm}}=2 \eta n c_{s}
$$

while the effect of the transverse ion flow is contained in the last term of (12), $\int_{0}^{L} d z \nabla \cdot J_{i \perp}=i k L n_{o} u_{x}$. Substituting these quantities in (12), we find

$$
e \phi=-\frac{i k L m_{e}}{2 \eta F_{e}\left(e \phi_{o}\right)} u_{x}
$$

For the case of a Maxwellian distribution, where equilibrium requires

$$
\frac{e \phi_{o}}{T_{e}}=\frac{1}{2} \ln \left(\frac{m_{i}}{2 \pi m_{e}}\right)
$$

and (38) reduces to

$$
e \phi=-(i k L / 2 \eta)\left(m_{i} T_{e}\right)^{1 / 2} u_{x} .
$$

To complete the calculation, we use the isothermal relation $P_{i}=n T_{i}$ and the perturbed ion continuity equation

$$
\frac{\partial n}{\partial t}+i k n_{o} u_{x}+\frac{\eta m c_{s}}{L}-\nu_{I} n=0
$$

where $\nu_{I}$ is the ionization rate. The last two terms of (41) cancel, since in equilibrium $\eta n_{o} c_{s}=\nu_{I} n_{o} L$. Using (40) and (41) in (35), we arrive at an equation for $u_{x}$

$$
\frac{\partial^{2} u_{x}}{\partial t^{2}}+\frac{k^{2} T_{i}}{m_{i}} u_{x}+\frac{k^{2} L c_{s}}{2 \eta} \frac{\partial u_{x}}{\partial t}+\nu_{i}^{\prime} \frac{\partial u_{x}}{\partial t}=0 .
$$

Each of the last three terms of (42) leads to a distinct type of response. The first term, in combination with the second term, leads to sound waves among the ions. These are ordinary sound waves associated with the ion pressure and propagating at the ion thermal speed, not the ion sound speed $c_{s}=\left(T_{e} / m_{i}\right)^{1 / 2}$. The last term of (42) represents ordinary collisional damping. The third term of (42), which arises from the response of the sheath potential, is the most interesting term. Taken together with the first term, the third term leads to diffusion of $u_{x}$, i.e., it is formally similar to a bulk viscosity term. The sheath potential thus leads to a dissipative effect which drives the flow toward uniformity of the velocity gradient $\partial u_{x} / \partial x$. In typical situations, this is the dominant term. To demonstrate this formally, consider the normal modes of (42)

$$
u_{x}(x, t)=u_{x}(0) e^{i k x+p t}
$$


where

$$
p=-\left(\frac{k^{2} L c_{s}}{4 \eta}+\frac{\nu_{i}^{\prime}}{2}\right) \pm\left[\left(\frac{k^{2} L c_{s}}{4 \eta}+\frac{\nu_{i}^{\prime}}{2}\right)^{2}-\frac{k^{2} T_{i}}{m_{i}}\right]^{1 / 2} .
$$

The modes are always damped, and if

$$
\frac{k L}{\eta}\left(\frac{T_{e}}{T_{i}}\right)^{1 / 2}+\frac{2 \nu_{i}^{\prime}}{k}\left(\frac{m_{i}}{T_{i}}\right)^{1 / 2}>4
$$

they are purely damped with no oscillation. Since $T_{e} / T_{i} \gg 1$, inequality (45) will normally be satisfied. For short wavelength modes, the dissipative effect arising from the electron sheath potential response easily dominates over the effect of ion pressure and quickly drives the system back to uniformity in transverse velocity.

In a simulation code, the time scale for this diffusive process $\tau_{\text {dif }}=2 \eta / k^{2} L c_{g}$ can be very short. In typical applications of our code, we may have $L \simeq 35 \mathrm{~cm}, c_{s} \simeq 3 \times 10^{5} \mathrm{~cm} / \mathrm{s}$, $\eta \simeq 0.3$, and the shortest wavelength modes have $\pi / k$ equal to the spacing of the field lines used in the grid, as small as $0.3 \mathrm{~cm}$. This gives $\tau_{\text {dif }}=5 \times 10^{-10}$. Since we wish to use time steps much longer than this, it is necessary to calculate the sheath potential implicitly, as discussed in Section VI.

Nonuniform Microwave Heating: It is important to note that the dissipative effect discussed in the preceding paragraph drives the system toward uniform transverse flux, not toward uniform density. This distinction has important consequences. Consider, for example, the "high mode" of an ECR discharge [51], in which all of the injected microwave energy is absorbed. The power density deposited into plasma heating thus depends only on the microwave flux, not on the plasma density or other details of the plasma state. The rate of creation of new electron-ion pairs by electron-impact ionization is then simply proportional to the microwave flux. Now suppose there is a small sinusoidal perturbation to the microwave flux, which leads to an ionization rate of the specified form $\dot{n}_{I 0}+\dot{n}_{I} e^{i k x}$. Then the plasma density will have a similar perturbed form $n_{o}+n e^{i k x}$. If there were no ion transport across field lines, the density perturbation would simply be proportional to the ionization source perturbation

$$
\frac{n}{n_{0}}=\frac{\dot{n}_{r}}{\dot{n}_{I 0}} .
$$

If, on the other hand, the ions can freely diffuse or flow across field lines, the nonuniformity in density will be smoothed out, i.e.,

$$
\frac{n}{n_{0}} \rightarrow 0
$$

To determine $n / n_{0}$ correctly, we use (35), (40), and the linearized continuity equation

$$
\frac{\partial n}{\partial t}+i k n_{o} u_{x}+\frac{\eta n c_{s}}{L}-\dot{n}_{I}=0
$$

(Note the difference in the last term on the LHS of (47), as compared to (41) where the ionization source is assumed to be isothermal.) Neglecting the effect of ion collisions, the steady state solution of (35), (40), and (47) is given by ${ }^{12}$

$$
\frac{n}{n_{0}}=\frac{\dot{n}_{I}}{\dot{n}_{I 0}} \frac{T_{e}}{T_{e}+2 T_{i}} .
$$

Comparing with (46a), we see that transverse ion transport does reduce the density perturbation, but only by a factor $T_{e} /\left(T_{e}+2 T_{i}\right)$ which is very close to unity. Diffusion toward uniformity in density is strongly inhibited by the sheath potential response.

Ion Transport to Vessel Walls: We have seen that the response of the sheath potential strongly suppresses internal cross-field flows within the plasma. However, it has been known for decades [48] that loss of ions to the walls, through nonambipolar cross-field flow, is an important process in conducting vessels. The sheath potential response can influence this flow, but it does not change the general conclusion. The effect of the sheath potential is merely to induce a smooth flow pattern which has uniform divergence, i.e., reduces the density at a comparable rate on different field lines.

A more systematic discussion of cross-field transport, for discharges within conducting vessels, will be provided in future publications.

\section{SimUlation OF AN ECR DisCHARGE IN AN INSULATING VESSEL}

An axisymmetric simulation code QUASI-rz has been developed which implements the formalism presented in Sections I-VI. Electrons are represented as particles transported along the field lines, as discussed in Section III. Each electron is characterized by its position $z$, its parallel velocity, and the value of its magnetic moment (which is equivalent to knowing the magnitude of its perpendicular velocity). Ions are represented as particles characterized by position in the $r-z$ plane and by all three components of velocity, as discussed in Section VI. Within the plasma, parallel electric fields are calculated as in Section III and transverse fields as in Section VI. Sheaths at passive surfaces are represented as thin potential barriers, as in Section IV, while the Bohm boundary condition is imposed as in Section V. Electron-neutral and ion-neutral collisions are included, via a Monte Carlo step (using the null-collision method [52], [53]) which occurs at the end of each particle push step. Electron-electron and ion-ion collisions are included in the Monte Carlo step via the recently developed Langevin formulation of multiple small-angle scattering [33], which is a great advance in efficiency over previous numerical formulations in terms of binary collisions.

The code is ultimately intended to provide a complete kinetic picture of an ECR discharge. We shall present here a sample calculation, which somewhat oversimplifies the representation of ECR experiments which are ongoing at NRL [54]-[60], but nonetheless illustrates a number of interesting

\footnotetext{
${ }^{12}$ The derivation of (40) assumed $T_{e}$ uniform, but this isothermal relation cannot hold exactly in the present circumstances. However, it is still permissable to use (40): the perturbations to $T_{e}$ are typically much smaller than those to $n$, because the ionization rate responds exponentially to $T_{e}$ but only linearly to $n$.
} 
points. The geometry is taken to be a cylindrical insulating vessel of length $35 \mathrm{~cm}$ and radius $7 \mathrm{~cm}$, representing the source region of the ECR, as illustrated in Fig. 2. The magnetic field configuration, as shown in Fig. 2, is the actual field applied in the experiment, ranging from $1 \mathrm{kG}$ at $z=0$ to $171 \mathrm{G}$ at $z=35 \mathrm{~cm}$. The gas is argon at pressure 1 mtorr, and a collision set is used which includes accurate cross sections for electron-neutral [61] and ion-neutral elastic scattering [37], electron-impact ionization and excitation [61], and ion-neutral charge exchange [37]-[39]. ${ }^{5}$ Leonhardt et al. [59] have recently shown that interactions between metastables and other components are not important in high-density $\mathrm{Ar}$ plasma at these pressures, so these are not included in the collision set.

The representation of electron cyclotron heating is simplified in the present simulation. We do not calculate the propagation of the microwaves or the details of their interaction with the electrons. Rather, we heat the electrons by giving each electron a random kick in transverse velocity every time it crosses the resonant surface, which lies in the plane $z=4 \mathrm{~cm}$. The magnitude of the kick $\Delta v_{\perp}$ is chosen randomly from a Maxwellian distribution $(2 \pi)^{-1 / 2} \exp \left[-\left(\Delta v_{\perp}\right)^{2} / 2 \Delta^{2}\right]$. The root-mean-square value $\Delta$ is chosen so that the total power absorbed by all the electrons on field line $j$ is equal to $P_{\mu}\left(r_{j, \text { res }}\right) A_{j \text {,res }}$, where $P_{\mu}(r)$ is the specified microwave power density, $r_{j, \text { res }}$ is the value of $r$ where field line $j$ intersects the resonant surface, and $A_{j, \text { res }}$ is the area of flux tube $j$ on the resonant surface. In the present simulation, we use

$$
P_{\mu}(r)=P_{o} \exp \left(-r^{2} / r_{o}^{2}\right)
$$

with $P_{o}=1.8 \mathrm{~W} / \mathrm{cm}^{2}$ and $r_{o}=7 \mathrm{~cm}$. The total absorbed microwave power, integrated from $r=0$ to the wall at $r=7$ $\mathrm{cm}$, is then $350 \mathrm{~W}$.

Given the simplication of the geometry and of the ECR heating process, one cannot necessarily expect the simulation to provide a quantitatively accurate picture of any particular experiment. However, the model includes all of the important effects that determine the plasma properties, including spatial variation of parameters, flow patterns, velocity distribution functions, and ionization fractions.

Simulation Results: We chose (arbitrarily) to initiate the simulation with uniform temperature $T_{e}=6.6 \mathrm{eV}$ and plasma density $n(r, z)$ proportional to $B(z, r)$ with $n=1.5 \times 10^{12}$ at $z=0$. After a time of order $300 \mu$ s the simulation evolves to a steady state, which appears to be insensitive to the initial conditions. We shall show graphics illustrating the steady state plasma at time $500 \mu \mathrm{s}$.

Fig. 5 shows the plasma density $n(r, z)$. Curiously, the peak is seen to be off axis, with a $20 \%$ density dip on axis, even though the microwave power is gently peaked on axis. As we shall see, this is a consequence of 2-D flows which differ from the field-aligned flow model of Section VII-A.

Fig. 6 shows surface plots of the ion fluid velocity components $u_{i z}(r, z)$ and $u_{i r}(r, z)$. We note that ions flow to all walls at flow velocity $u_{i}^{\perp} \simeq c_{s}$ in accordance with the Bohm condition. At every wall, a presheath is evident in Figs. 5 and 6 , wherein the ions are accelerated to $c_{s}$, but the presheath is

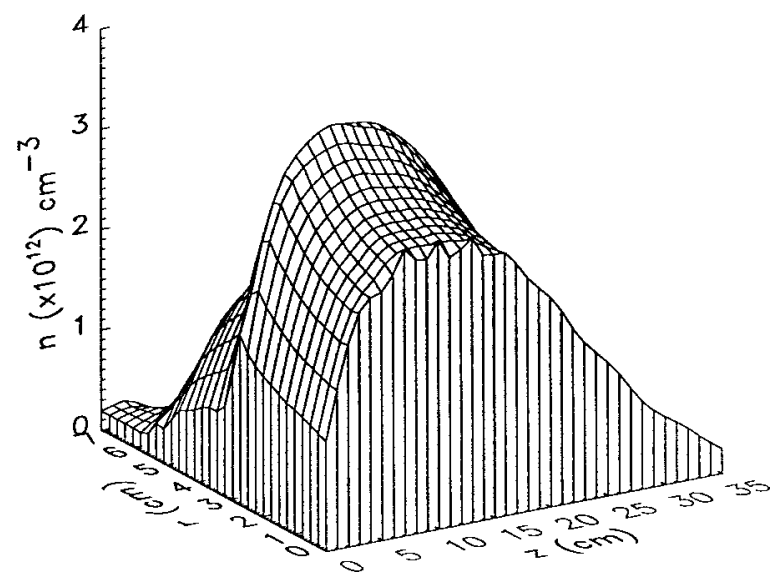

(a)

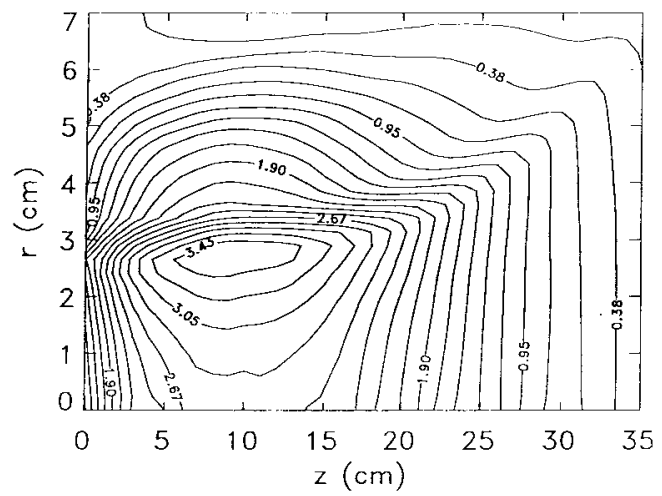

(b)

Fig. 5. Plasma density $n(z, r) \times 10^{12} \mathrm{~cm}^{-3}$ shown as (a) a surface plot and (b) a contour plot, at time $500 \mu \mathrm{s}$, taken from a QUASI-rz simulation with Ar pressure $1 \mathrm{mT}$, microwave power $350 \mathrm{~W}$, and insulating vessel walls.

minimal on the downstream end wall where the acceleration largely occurs as a consequence of magnetic field expansion. Fig. 7 is a vector plot of the ion flux, which more clearly illustrates the surprisingly complex nature of the flow. The important feature is that the outer field lines of Fig. 2, which intersect the radial wall at an acute angle of $5-19^{\circ}$, lose ions to the wall over a relatively large area at perpendicular velocity $u_{i}^{\perp}=c_{s}$. This loss of ions is not resupplied through flow along the field line, as in the model of Section VII-A, but mainly through cross-field flow from adjacent field lines. However, the quasi-neutrality condition specifies that no field line can have a net gain or loss of ions through cross-field flow within the plasma. Thus, there must be an inward return flow upstream. Comparing Fig. 7 with Fig. 2, it can be seen that the result is a flow pattern which is fairly close to field aligned in the downstream region and yet shows a significant cross-field eddy flow structure.

The nature of the flow has a significant impact on the density and temperature profile within the plasma. The cross-field flow permits the rate of ion loss from those field lines that terminate obliquely on the radial wall to be greater than the loss rate from the interior field lines that terminate nearly normally on the end wall. (Recall that in the 1-D model of Section VII-A, the loss rate depends only on the length of the field line, but not on the angle at which the field line intersects the wall.) Thus there is 


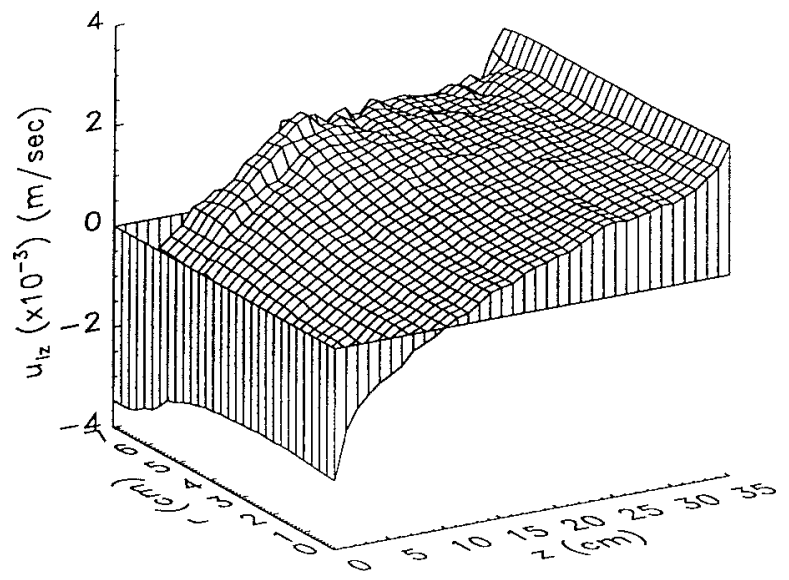

(a)

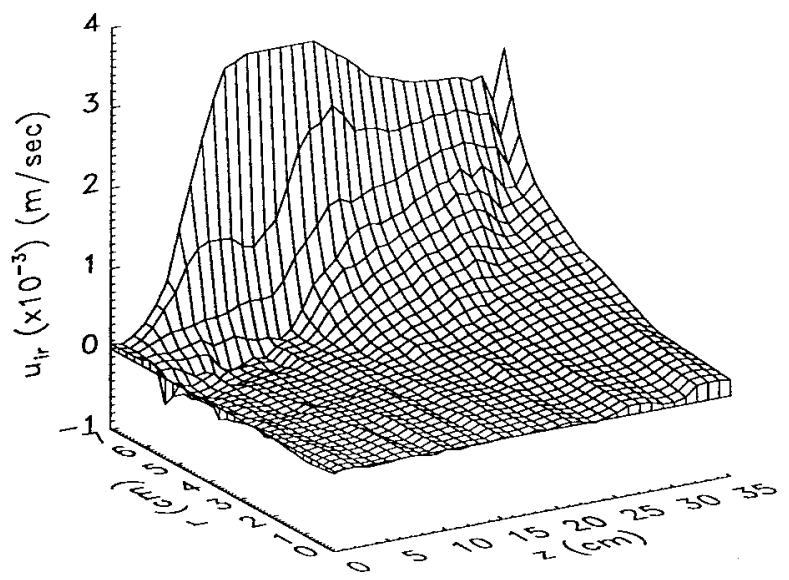

(b)

Fig. 6. Surface plot of the ion fluid velocity components (a) $u_{i z}$ and (b) $u_{i r}$ at time $500 \mu \mathrm{s}$, from the simulation of Fig. 5 .

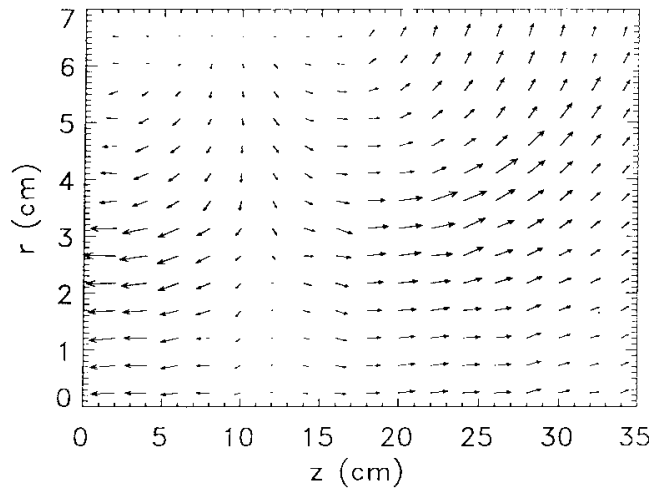

Fig. 7. Vector plot of the ion flux at time $500 \mu \mathrm{s}$, from the simulation of Fig. 5. Note that the aspect ratio of the plot is different from that of the simulation.

an abrupt decrease in the plasma density, as we move from the last field line that terminates on the end wall $(j=9)$ to the first field line that terminates on the radial wall. This is evident in Fig. 5. In Fig. 8 we plot the average value of the density $\left\langle n_{j}\right\rangle$ on field line $j$ as a function of $j$. This figure shows even more clearly the sharp change in the plasma around field line $j=9$. As $j$ increases further, i.e., as we consider the outer

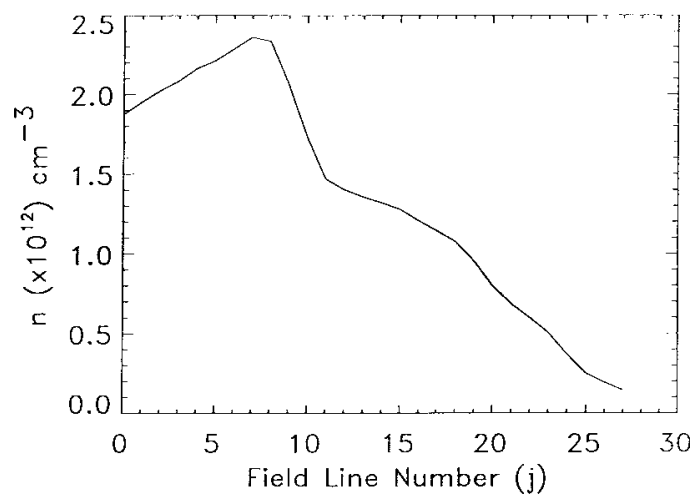

Fig. 8. The average density on field line $j$ at time $500 \mu \mathrm{s}$, as a function of the field line number.

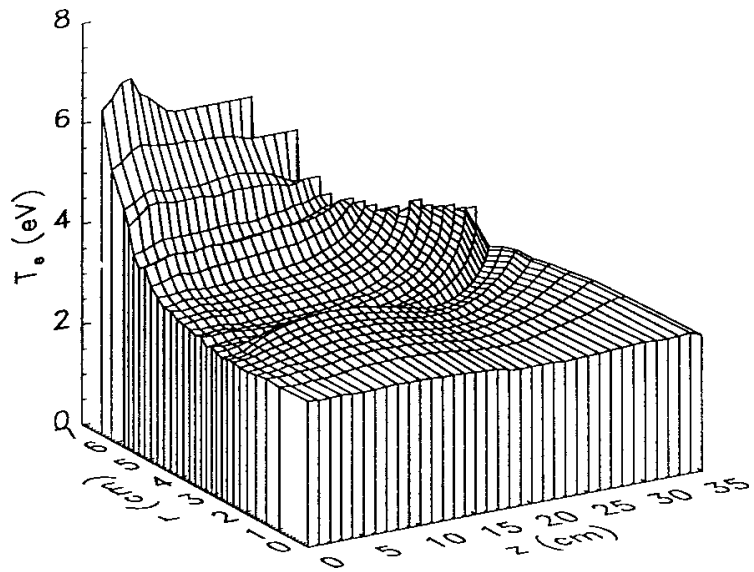

Fig. 9. Surface plot of electron temperature $T_{e}$, defined as $\left\langle m_{e} v^{2} / 3\right\rangle$ at time $500 \mu \mathrm{s}$

field lines that terminate at decreasing values of $z$, ions are lost still faster and there are further decreases in $\left\langle n_{j}\right\rangle$.

The electron temperature $T_{e}$, which is found to be quite close to isotropic, is shown as a surface plot in Fig. 9. $T_{e}$ is close to flat, as expected, on the inner field lines $j \leq 9$. On field lines $j>9$, which terminate on the radial wall, $T_{e}$ increases as the field line becomes shorter. This is a consequence of the more rapid loss of ions on these short field lines, as discussed in Section VII-A. However, the electron temperature, which we define as the mean $\left\langle m_{e} v^{2} / 3\right\rangle$, is found to be significantly larger than the values obtained from the fluid model of Section VII-A. The primary reason for this is that the electron energy distribution function falls off from Maxwellian in the high energy regime, which controls electron-impact ionization. Thus $T_{e}$ must be somewhat larger to give the appropriate ionization rate. Distribution functions will be discussed in more detail in forthcoming work. This type of temperature and density pattern is seen in the NRL experiments [58].

The cross-field ion flow also leads to a rather subtle effect on the density of the interior field lines. At large $z$ (downstream), the flow is divergent and depletes the ions, but in the region of highest density near $z=10 \mathrm{~cm}$, the flow is convergent and brings in additional ions. Thus this flow tends to increase the 


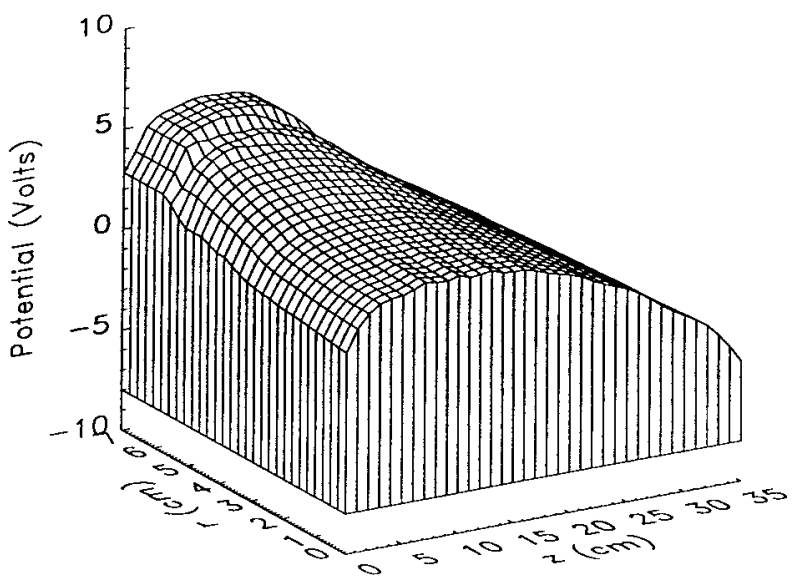

(a)

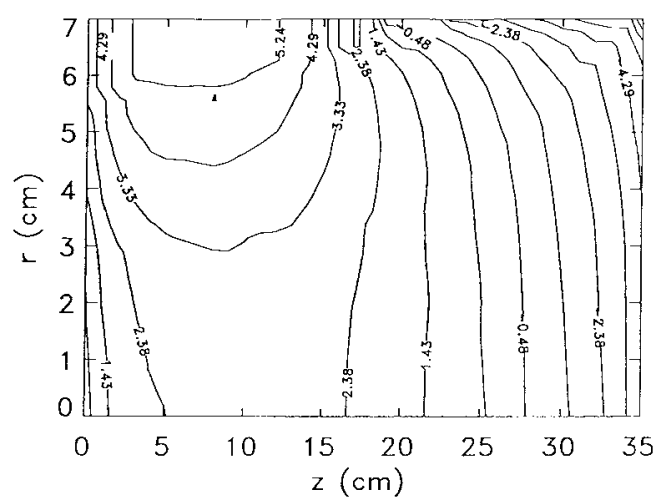

(b)

Fig. 10. Plasma potential $\phi(r, z)$ from the simulation of Fig. 5 at time 500 $\mu \mathrm{s}$, shown as (a) a surface plot and (b) a contour plot.

peakedness of the density profile $n_{j}(z)$ along a field line, i.e., to increase the maximum of $n_{j}$ but reduce $n_{j}$ at the ends of the field line. Fig. 7 shows that the cross-field eddy flow is strongest on the field lines $(j=7,8,9)$ that terminate at the outside of the end wall. On field lines that are near the axis, the flow is very nearly field-aligned. Thus the density profile $n_{j}(z)$ is more peaked on field lines $j=7,8,9$ than on the inner field lines near the axis. However, the loss rate of ions to the walls is proportional to the density at the end of the field line, and thus is slower on field lines $j=7,8,9$. Applying the arguments of Section VII-A in this more general multidimensional context, reducing the particle loss rate increases the density all along the field line. This is the explanation of the off-axis density peak which can be seen in Fig. 5 at $z=8, r=2.8$, and which is also evident in Fig. 8.

The potential profile $\phi(r, z)$, shown in Fig. 10, is more complex than might have been anticipated. It is not peaked on axis, and it is not simply indicative of the electron pressure profile, as in an unmagnetized plasma. Rather, $\phi(r, z)$ has a saddle on the axis at $z=9$ and for $z \lesssim 15 \mathrm{~cm} \phi$ is a monotonically increasing function of $r$, except in the presheath at the radial wall. Downstream, $\phi$ is a monotonically decreasing function of $r$. This structure is just what is needed to drive the radially outward ion flow downstream and the inward return flow upstream. This type of potential structure has been observed in the NRL experiments [58].

\section{CONCLuding Remarks}

We have presented a variety of techniques for modeling the quasi-neutral region of a plasma discharge. These include methods for: 1) determining the electric field parallel to $B$ and the associated electron transport; 2) determining the potential variations transverse to $B$ and the associated ion transport; 3 ) determining the spatially and temporally dependent sheath potential; and 4) enforcing the Bohm flow condition. All of these techniques are time dependent, fully kinetic, and based on PIC modeling of both the electrons and ions. In all cases, the fields and potentials are determined directly from the requirement of quasi-neutrality. Poisson's equation is not used, the debye shielding length is effectively set to zero, and electron plasma oscillations are not present in the modeling. As a result, the models focus on the macroscopic processes that are actually of interest and permit the use of large time steps and spatial gridding that enhance computational efficiency.

The modeling techniques have been discussed in the context of magnetized ECR discharges for processing applications, but we believe that they lend themselves to a wide range of plasma conditions, including unmagnetized plasmas, unbounded plasmas, and high-temperature collisionless plasmas of interest in fusion and space physics.

The power of the techniques has been demonstrated by applying them analytically to some simple fluid situations and by incorporating them in a 2-D (axisymmetric) PIC simulation model for an ECR discharge. Analytic study of a discharge contained within a conducting vessel revealed significant modifications to the classic Simon diffusion across a magnetic field [48]-[50]. Simulation of an ECR discharge contained within insulating walls revealed unanticipated and important structural features which are dependent on 2-D ion flows.

In this paper we have not discussed the modeling of the microwave-plasma interaction. Computationally efficient approaches to this problem will be discussed in subsequent publications. Simulations of discharges within conducting vessels are also in progress and will be reported in future publications.

\section{APPENDIX}

Consider the electrons to be guiding center particles attached to a particular field line in the $r-z$ plane. The phase space variables necessary to characterize these electrons are then $\zeta$, $v_{\|}$, and $\mu$, defined in Section III. We can define an electron distribution function $F\left(\zeta, v_{\|}, \mu\right)$ in this three-dimensional (3D) phase space, and the corresponding Boltzmann equation is

$$
\frac{\partial F}{\partial t}+v_{\|} \frac{\partial F}{\partial \zeta}-\left(\frac{e E_{\|}}{m_{e}}+\frac{\mu}{m_{e}} \frac{\partial B}{\partial \zeta}\right) \frac{\partial F}{\partial v_{\|}}=K
$$

where $B$ is the magnitude of the magnetic field, $K$ is the collision integral, and the second term in parentheses on the left-hand side is the mirror force [23]. Since the area of a flux tube is inversely proportional to $B$, the distribution function $f\left(\boldsymbol{r}, v_{\|}, \mu\right)$ in 3-D spatial coordinates $\boldsymbol{r}$ can be related to 
$F\left(\zeta, v_{\|}, \mu\right)$ as

$$
f\left(\boldsymbol{r}, v_{\|}, \mu\right)=B F\left(\zeta, v_{\|}, \mu\right) .
$$

Substituting (A2) in (A1), the Boltzmann equation becomes

$$
\frac{\partial}{\partial t} \frac{f}{B}+v_{\|} \frac{\partial}{\partial \zeta} \frac{f}{B}-\left(\frac{e E_{\|}}{m_{e}}+\frac{\mu}{m_{e}} \frac{\partial B}{\partial \zeta}\right) \frac{\partial}{\partial v_{\|}} \frac{f}{B}=K .
$$

Since $B$ depends only on spatial location, (A3) can be rewritten as

$$
\frac{\partial f}{\partial t}+v_{\|} B \frac{\partial}{\partial \zeta} \frac{f}{B}-\left(\frac{e E_{\|}}{m_{e}}+\frac{\mu}{m_{e}} \frac{\partial B}{\partial \zeta}\right) \frac{\partial f}{\partial v_{\|}}=K B .
$$

Now, taking the moment $\int d v_{\|} m_{e} v_{\|}$of (A4), we arrive at (4) of the main text

$$
\begin{array}{r}
\frac{\partial}{\partial t}\left(n_{e} m_{e} u_{e \|}\right)+B \frac{\partial}{\partial \zeta} \frac{\left(P_{e \|}+n_{e} m_{e} u_{e \|}^{2}\right)}{B} \\
+\bar{\mu} n_{e} \frac{\partial B}{\partial \zeta}+n e E_{\|}=-\nu_{e} n_{e} m_{e} u_{e \|}
\end{array}
$$

where the friction term is

$$
-\nu_{e} n_{e} m_{e} u_{e \|} \equiv \int d v_{\|} m_{e} v_{\|} K B
$$

Note that

$$
\bar{\mu} \equiv P_{e \perp} / n_{e} B
$$

so that the momentum conservation equation (A5) can also be written

$$
\begin{aligned}
& \frac{\partial}{\partial t}\left(n_{e} m_{e} u_{e \|}\right)+\frac{\partial}{\partial \zeta}\left(P_{e \|}+n_{e} m_{e} u_{e \|}^{2}\right) \\
& \quad+\frac{P_{e \perp}-P_{e \|}-n_{e} m_{e} u_{e \|}^{2}}{B} \frac{\partial B}{\partial \zeta}+n e E_{\|}=-\nu_{e} n_{e} m_{e} u_{e \|}
\end{aligned}
$$

which shows that the mirror force vanishes from the fluid equation when the electron velocity distribution is isotropic.

\section{ACKNOWLEDGMENT}

The authors especially acknowledge contributions by R. F. Fernsler to their understanding of collisional processes and many other matters. They appreciate the stimulation provided by interactions with their experimental colleagues, including S. Douglass, D. Hinshelwood, C. R. Eddy, V. Shamamian, D. Leonhardt, J. E. Butler, B. Weber, and others. They are also grateful to I. Haber for many good ideas about numerical techniques and to N. Hershkowitz for sharing his insight.

\section{REFERENCES}

[1] M. A. Lieberman and A. J. Lichtenberg, Principles of Plasma Discharges and Materials Processing. New York: Wiley-Interscience, 1994.

[2] C. K. Birdsall, "Particle-in-cell charged particle simulations, plus Monte Carlo collisions with neutral atoms, PIC-MCC," IEEE Trans. Plasma Sci., vol. 19, pp. 65-85, Apr. 1991.

[3] R. W. Boswell and I. J. Morey, "Self-consistent simulation of a parallelplate RF discharge," Appl. Phys. Lett., vol. 52, pp. 21-23, Jan. 1988.

[4] D. Vender and R. W. Boswell, "Numerical modeling of low pressure RF plasmas," IEEE Trans. Plasma Sci., vol. 18, pp. 725-732, Aug. 1990.
[5] M. Surendra, D. B. Graves, and I. J. Morey, "Electron heating in low pressure RF glow discharges," Appl. Phys. Letters, vol. 56, pp. 1022-1024, Mar. 12, 1990.

[6] M. M. Turner and M. B. Hopkins, "Anomalous sheath heating in low pressure RF discharge in nitrogen," Phys. Rev. Lett., vol. 69, pp. 3511-3514, Dec. 1992.

[7] V. Vahedi, C. K Birdsall, M. A. Lieberman, G. DiPeso, and T. D. Rognlien, "Capacitive RF discharges modeled by particle-in-cell Monte Carlo simulation. 1: Analysis of numerical techniques," Plasma Sources Sci. Technol., vol. 2, pp. 261-272, Nov. 1993.

[8] R. K. Porteus and D. B. Graves, "Modeling and simulation of magnetically confined low pressure plasmas in two dimensions," IEEE Trans. Plasma Sci., vol. 19, pp. 204-213, Apr. 1991.

[9] D. B. Graves, H. Wu, and R. K. Porteus, "Modeling and simulation of high density plasmas," Jpn. J. Appl. Phys., vol. 32, pp. 2999-3006, June 1993.

[10] R. K. Porteus, H. Wu, and D. B. Graves, Plasma Sources Sci. Technol., vol. 3, pp. 25-39, 1994.

[11] K. A. Ashtiani, J. L Shohet, W. N. G. Hitchon, G.-H. Kim, and N. Hershkowitz, "A 2-D particle-in-cell simulation of an electron-cyclotronresonance etching tool," J. Appl. Phys., vol. 78, pp. 2270-2278, Aug. 1995.

[12] V. P. Gopinath and T. A. Grotjohn, "Three-dimensional electromagnetic PIC model of a compact plasma source," IEEE Trans. Plasma Sci., vol. 23, pp. 602-608, Aug. 1995.

[13] J. Denavit, "Time-filtering particle simulations with $\omega_{p} \Delta t \gg 1$," $J$. Comput. Phys., vol. 42, pp. 337-366 July 1981

[14] R. J. Mason, "Implicit moment particle simulation of plasmas," $J$. Comput. Phys., vol. 41, pp. 233-244, June 1981.

[15] A. Friedman, A. B. Langdon, and B. I. Cohen, "A direct method for implicit particle-in-cell simulations," Comments Plasma Phys. Controlled Fusion, vol. 6, pp. 225-236, 1981.

[16] A. B. Langdon, B. I. Cohen, and A. Friedman, "Direct implicit large time-step particle simulation in plasmas," J. Comput. Phys., vol. 51, pp. 107-138, July 1983.

[17] J. U. Brackbill and B. I. Cohen, Eds., Multiple Time Scales. New York: Academic, 1985, ch. 8, 9, 11.

[18] D. W. Hewett and D. J. Lawson, "Solution of simultaneous partial differential equations using dynamic ADI: Solution of the streamlined Darwin field equations," J. Comput. Phys., vol. 101, pp. 11-24, July 1992.

[19] M. R. Gibbons and D. W. Hewett, "Characterization of the Darwin direct implicit particle-in-cell method and resulting guidelines for operation," J. Comp. Phys., vol. 130, pp. 54-66, Jan. 1997.

[20] B. I. Cohen, A. B. Langdon, D. W. Hewett, and R. J. Procassini, "Performance and optimization of direct implicit particle simulation," J. Comput. Phys., vol. 81, pp. 151-168, Mar. 1989.

[21] A. B. Langdon, "Kinetic theory for fluctuations and noise," Phys. Fluids, vol. 22, pp. 163-171, Jan. 1979.

[22] S. E. Parker, A. Friedman, S. L. Ray, and C. K. Birdsall, "Bounded multiscale plasma simulation: Application to sheath problems," J. Comput. Phys., vol. 107, pp. 388-402, Aug. 1993.

[23] F. F. Chen, Introduction to Plasma Physics. New York: Plenum, 1974

[24] A. G. Sgro and C. W. Nielson, "Hybrid model studies of ion dynamics and magnetic diffusion during pinch implosions," Phys. Fluids, vol. 19, pp. 126-133, Jan. 1976.

[25] D. W. Hewett and C. W. Nielson, "A multidimensional quasi-neutral plasma simulation model,” J. Comput. Phys., vol. 29, pp. 219-236, Nov. 1978.

[26] J. A. Byers, B. I. Cohen, W. C. Condit, and J. N. Hanson, "Hybrid simulation of quasi-neutral phenomena in a magnetized plasma," $J$ Comput. Phys., vol. 27, pp. 363-396, June 1978.

[27] H. Okuda, J. M. Dawson, A. T. Lin, and C. C. Lin, "Quasi-neutral particle simulation model with application to ion wave propagation," Phys. Fluids, vol. 21, pp. 476-482, Mar. 1978.

[28] J. K. Lee and C. K. Birdsall, "Velocity space ring-plasma instability, magnetized, Part 1: Theory," Phys. Fluids, vol. 22, pp. 1306-1314, July 1979.

[29] D. W. Hewett, "A global method of solving electron-field equations in a zero-inertia-electron-hybrid plasma simulation code," J. Comput. Phys., vol. 38, pp. 378-395, Dec. 1980.

[30] D. S. Harned, "Quasi-neutral hybrid simulation of macroscopic plasma phenomena," J. Comput. Phys., vol. 47, pp. 452-462, Sept. 1982.

[31] R. J. Mason, "Hybrid and collisional implicit plasma simulation models," in Multiple Time Scales, J. U. Brackbill and B. I. Cohen, Eds. New York: Academic, 1985, ch. 8.

[32] D. W. Hewett, "Low-frequency electromagnetic (Darwin) applications in plasma simulation," Comput. Phys. Comm., vol. 84, pp. 243-247, Nov. 1994. 
[33] W. M. Manheimer, M. Lampe, and G. Joyce, "Langevin representation of Coulomb collisions in PIC simulations," J. Comput. Phys., vol. 138 pp. 563-584, Dec. 1997.

[34] W. M. Manheimer, "A simple scheme for implementing wave absorption in quasi-neutral PIC simulations of ECR plasma," Naval Research Laboratory, Washington, DC, Memo. Rep. NRL/MR/6707-98-8122, 1998.

[35] L. S. Frost and A. V. Phelps, "Momentum-transfer cross sections for slow electrons in He, Ar, Kr," Phys. Rev. A, vol. 136, pp. 1538-1545, Dec. 1964.

[36] L. G. Christophorou, Atomic and Molecular Radiation Physics. New York: Wiley-Interscience, 1970.

[37] A. V. Phelps, "Cross sections and swarm coefficients for nitrogen ions and neutrals in $\mathrm{N}_{2}$ and argon ions and neutrals in Ar for energies from $0.1 \mathrm{eV}$ to $10 \mathrm{keV}$," J. Phys. Chem. Ref. Data, vol. 20, no. 3, pp. 557-573, 1991.

[38] M. A. Lieberman and A. J. Lichtenberg, Principles of Plasma Discharges and Materials Processing. New York: Wiley-Interscience, 1994, p. 78

[39] M. L. Vestal, C. R. Blakley, and J. H. Futrell, "Crossed-beam measurements of differential cross sections for elastic scattering and charge exchange in low energy $\mathrm{Ar}^{+}-\mathrm{Ar}$ collisions," Phys. Rev. A, vol. 17, pp. 1337-1342, Apr. 1978.

[40] M. Lampe, G. Joyce, and W. M. Manheimer, "Quasi-neutral kinetic model of magnetized plasma discharges," in Lectures in Plasma Physics and Technology, V. Stefan, Ed. San Diego, CA: Institute for Advanced Physics Studies Press, 1999.

[41] G. Joyce, M. Lampe, and W. M. Manheimer, "Electrostatic particle-incell simulation technique for quasi-neutral plasma," J. Comput. Phys., vol. 138, pp. 540-562, Dec. 1997.

[42] S. E. Parker, R. J. Procassini, C. K. Birdsall, and B. I. Cohen, "A Suitable boundary condition for bounded plasma simulation without sheath resolution," J. Comput. Phys., vol. 104, pp. 41-49, Jan. 1993.

[43] K.-U. Riemann, "The Bohm criterion and sheath formation," J. Phys. D, Appl. Phys., vol. 24, pp. 493-518, Apr. 1991.

[44] _ "Kinetic theory of the plasma sheath in a weakly ionized plasma," Phys. Fluids, vol. 24, pp. 2163-2172, Dec. 1981.

[45] V. A. Godyak, Soviet Radio Frequency Discharge Research. Falls Church, VA: Delphic Assoc., 1986.

[46] M. A. Lieberman and A. J. Lichtenberg, Principles of Plasma Discharges and Materials Processing. New York: Wiley-Interscience, 1994, p. 137.

[47] G.-H. Kim, N. Hershkowitz, D. A. Diebold, and M.-H. Cho, "Magnetic and collisional effects on presheaths," Phys. Plasmas, vol 2, pp. 3222-3233, Aug. 1995.

[48] A. Simon, "Ambipolar diffusion in a magnetic field," Phys. Rev., vol. 98, pp. 317-318, 1955

[49] _ An Introduction to Thermonuclear Research. New York: Pergamon, 1959.

[50] M. A. Lieberman and A. J. Lichtenberg, Principles of Plasma Discharges and Materials Processing. New York: Wiley-Interscience, 1994, pp. 140-145.

[51] D. A. Carl, M. C. Williamson, M. A. Lieberman, and A. J. Lichtenberg, "Axial radio frequency electric field intensity and ion density during low to high mode transition in argon electron cyclotron frequency discharges," J. Vac. Sci. Technol. B, Microelectron. Process Phenom., vol. 9, pp. 339-347, Mar. 1991.

[52] J. P. Boeuf and E. Marode, "A Monte Carlo analysis of an electron swarm in a nonuniform field: The cathode region of a glow discharge in helium," J. Phys. D, vol 15, pp. 2169-2187, Nov. 1982

[53] S. L. Lin and J. N. Bardsley, "Monte Carlo simulations of ion motion in drift tubes," J. Chem. Phys., vol. 66, pp. 435-445, Jan. 1977.

[54] S. R. Douglass, C. R. Eddy, Jr., and B. V. Weber, "Faraday rotation of microwave fields in an electron cyclotron resonance plasma," IEEE Trans. Plasma Sci., vol. 24, pp. 16-17, Feb. 1996.

[55] G. Mehlman, C. R. Eddy, Jr., and S. R. Douglass, "Characterization of electron cyclotron resonance plasmas by vacuum ultraviolet spectroscopy," J. Appl. Phys., vol. 78, pp. 6421-6426, Dec. 1995.

[56] D. Leonhardt, C. R. Eddy, V. A. Shamamian, R. T. Holm, O. J. Glembocki, and J. E. Butler, "Sorface chemistry and damage in highdensity plasma etching of gallium arsenide," J. Vac. Sci. Technol. A, vol. 16, pp. 1547-1551, May/June 1998.
[57] C. R. Eddy, Jr., O. J. Glembocki, D. Leonhardt, V. A. Shamamian, R T. Holm, B. D. Thoms, J. E. Butler, and S. W. Pang, "Gallium arsenide surface chemistry and surface damage in a chlorine high density etch process," J. Electron. Mater., vol. 26, pp. 1320-1325, Nov. 1997.

[58] C. R. Eddy, Jr., D. Leonhardt, S. R. Douglass, B. D. Thoms, V. A Shamamian, and J. E. Butler, "Characterization of $\mathrm{Cl}_{2} / \mathrm{Ar}$ high density plasmas for semiconductor processing," J. Vac. Sci. Technol. A, Vac. Surf. Films, to be published.

[59] D. Leonhardt, C. R. Eddy, Jr., V. A. Shamamian, R. F. Fernsler, and J. E. Butler, "Argon metastables in a high density processing plasma," $J$. Appl. Phys., vol. 83, pp. 2971-2979, Mar. 1998.

[60] D. Leonhardt, C. R. Eddy, Jr., V. A Shamamian, R. T. Holm, O. J. Glembocki, B. D. Thoms, J. E. Butler, and S. W. Pang, "Ion energy effects on surface chemistry and damage in a high density plasma etch process for GaAs," Jpn. J. Appl. Phys. (Part 2), vol. 37, pp. 577-579, May 1998.

[61] K. Tachibana, "Excitation of the $1 \mathrm{~S}_{5}, 1 \mathrm{~S}_{4}, 1 \mathrm{~S}_{3}$, and $1 \mathrm{~S}_{2}$ levels of argon by low-energy electrons," Phys. Rev. A, vol. 34, pp. 1007-1015, Aug. 1986.

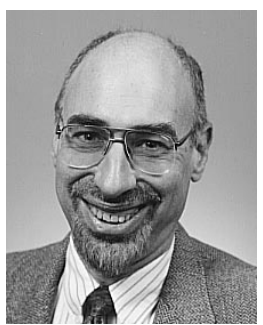

Martin Lampe (M'88) was born in Brooklyn, NY He received the A.B. degree from Harvard College, Cambridge, MA, and the M.A. and Ph.D. degrees in physics from the University of California, Berkeley.

After spending two years at New York University, he joined the Naval Research Laboratory, Washington, DC, in 1969. He has worked on a variety of topics in plasma theory, development of numerical techniques, physics of electron and ion beams, and currently on plasma processing.

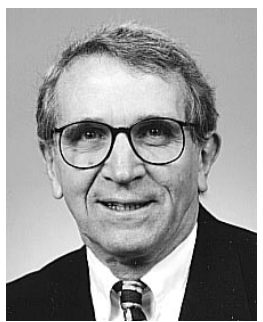

Glenn Joyce (M'95) received the Ph.D. degree in physics from the University of Missouri, Columbia, in 1966.

He was on the faculty of the University of Iowa, Iowa City, from 1967 until 1981. He has been a Senior Research Physicist at the Naval Research Laboratory, Washington, DC, since 1981. His current research interests include ionospheric modeling and plasma processing.

Wallace M. Manheimer (M'85-SM'86-F'93), for a photograph and biography, see p. 1296 of the August 1998 issue of this TRANSACTIONS.

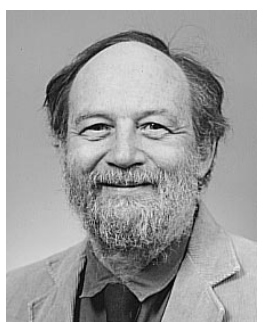

Steven P. Slinker received the Ph.D. degree in mathematical physics from Indiana University, Bloomington, in 1975 .

He has worked in the Plasma Physics Division of the Naval Research Laboratory, Washington, DC since 1985. His current research interests involve space weather prediction and numerical modeling of the earth's magnetosphere, as well as plasma processing. 